\title{
Physicochemical Properties of Dried Ginseng Powder Manufactured using Different Roasting Pretreatments and Cryogenic Milling conditions
}

\author{
Hayeong Jeong, Dong Hyeon Park, Han Geuk Seo, Mi-Jung Choi and Youngjae Cho* \\ Department of Food Science and Biotechnology of Animal Resources, Konkuk University, 120 Neungdong- \\ ro, Gwangjin-gu, Seoul 05029, Korea \\ *Correspondence: E-mail address: moonjae@konkuk.ac.kr Tel.: +82-2-450-3048. Fax: +82-02-455-3726.
}

\begin{abstract}
This study was aimed at investigating the effects of roasting and cryogenic milling on particle size, water solubility, and amount of bioactive components in ginseng root. Samples were pulverized by cryogenic milling, and one treatment condition was selected for each size range (10$50 \mu \mathrm{m}$, and $>50 \mu \mathrm{m})$. The selected samples were roasted at different temperatures $\left(160-200{ }^{\circ} \mathrm{C}\right)$ followed by cryogenic milling. Powdered samples were analyzed for their physicochemical characteristics. Results revealed that roasted samples exhibited significantly smaller particle size than controls (not roasted, $p<0.05$ ), and the particle size of roasted samples decreased with increase in roasting temperature until flocculation occurred around $180-190^{\circ} \mathrm{C}$. With decrease in particle size of ginseng; water solubility index, antioxidant activity, total polyphenol content, and total polysaccharide content were observed to increase. Ginseng samples showed an increasing trend in antioxidant activity with decreasing particle size $(p>0.05)$ with significantly higher values for all roasted samples compared to the controls $(p<0.05)$. Non-polar ginsenosides such as $\operatorname{Rg} 2(\mathrm{~S}), \operatorname{Rg} 2(\mathrm{R})$, Rg3(S), Rg3(R), and Rh1(S) showed an increase in temperature-dependent manner. This study revealed that roasting at certain temperature range with cryogenic milling has positive effects on ginseng by reducing its particle size, and increasing water solubility and bioactive components.
\end{abstract}

Keywords: bioactive components, cryogenic milling, ginseng, particle size, roasting, water solubility

\section{Introduction}

Ginseng (Panax ginseng C.A. Meyer) is widely used in herbal medicine and has served as a functional food for over 2,000 years in oriental countries [1]. The use of ginseng root has been extensively preferred due to the presence of pesticide residues in ginseng leaf and stem [2]. Ginseng roots contain many bioactive components such as ginsenosides, phenolic compounds, acidic polysaccharides, and free amino acids that provide diverse health benefits to humans [3-5]. Specifically, major ginsenosides ( $\mathrm{Rb} 1, \mathrm{Rb} 2, \mathrm{Rb} 3, \mathrm{Rc}, \mathrm{Rd}, \mathrm{Re}, \mathrm{Rf}$, and $\mathrm{Rg} 1$ ) in white ginseng roots have antioxidant, anti-cancer, anti-stress, anti-fatigue, and anti-inflammatory effects, and are involved in enhancing immunity, regulating blood pressure, improving brain function, and maintaining homeostasis [6-8]. Due to the presence of these bioactive compounds, fresh ginseng has a variety of benefits; however, high moisture content (approximately 70\%) in fresh ginseng provides appropriate conditions for microbial growth, and enzymatic and non-enzymatic reactions. This results in rapid degradation in quality and reduction in medicinal efficacy of ginseng roots, and thereby, depreciation in the commercial value of ginseng [9]. Therefore, dried ginseng with relatively low moisture content (approximately $<15 \%$ ) can be stored for the long-term. Furthermore, dried ginseng is easy to pack, ship, and store because of its reduced weight and volume [10,11]. At present, dried ginseng products 
primarily exist as an extract or in powdered forms in global herbal markets [12]. Although more products based on ginseng extract have been launched than the powdered form, a study has shown that hydrophobic and amphiphilic components cannot be effectively extracted from ginseng [12]. However, the powdered form has been reported to contain all the hydrophilic, hydrophobic, and amphiphilic bioactive components of ginseng [12]. The use of powdered ginseng in beverages is limited due to its low solubility. Thus, it is necessary to improve the solubility of ginseng powder.

Roasting is a suitable pretreatment that can enhance the solubility of ginseng powder. Pretreatment methods such as roasting result in decomposition, synthesis, and condensation reactions due to heat, which alters the constituent material facilitating the dissolution of the components of food [13]. Thus, there are a variety of reports showing that roasting increases ginsenoside as well as soluble solid content of ginseng [14,15]. Additionally, roasting promotes Maillard reaction between the reducing sugar (carbonyl group) and nitrogen compound (amino group), which generates a browning substance, melanoidin [16,17]. Furthermore, these melanoidins promote the antioxidant activity, and enhance the color and flavor of the product [16,17]. In addition to pretreatment, this study evaluated cryogenic milling of ginseng, which is not a common commercial milling method, and to our knowledge, we are the first to use such a method on ginseng. Conventionally, powderization technology uses high-temperature milling machines such as hammer mill, pin mill, ball mill, and air classifying mill. However, high-temperature milling affects ginseng quality through processes such as destruction of bioactive components and reduction of moisture content due to the generation of frictional heat through milling. However, low-temperature milling preserves the bioactive components and protects them from heat damage. Additionally, lowtemperature milling has been shown to transform the cell wall layers of ginseng into brittle material, thereby rapidly reducing the powder size and limiting re-aggregation, ultimately resulting in increased solubility [18]. A previous study on relationship between particle size and water solubility has revealed that with decreasing particle size, the components (carbohydrate, protein, and lipid) of ginseng dissolve efficiently [19]. Only few studies on ginseng grinding with low temperature milling have been published so far. Furthermore, study on ginseng using cryogenic (ultra-low temperature) milling has never been reported. Such cryogenic milling at ultra-low temperature can produce ginseng powder of smaller size and may help in increasing solubility more than what is obtained using low temperature milling [20,21].

Thus, the purpose of this study was to evaluate the effects on roasting and cryogenic milling at ultra-low temperature $\left(-196^{\circ} \mathrm{C}\right)$ on particle size, water solubility and bioactive components in ginseng root. Furthermore, experiments were conducted to compare the physicochemical characteristics such as particle size, appearance, color, water solubility index (WSI), antioxidant activity, total polysaccharide content, acidic polysaccharide content, and ginsenosides based on particle size.

\section{Materials and Methods}

\subsection{Ginseng preparation and pretreatment}

White dried ginseng (4-year-old) was purchased from Korea bio red ginseng market (Geumsan, Korea). Ginseng roots were cut into section of approximately $50 \mathrm{~mm}$ in length. Before grinding, dried ginseng $(125 \mathrm{~g})$ was roasted at $160{ }^{\circ} \mathrm{C}, 170{ }^{\circ} \mathrm{C}, 180{ }^{\circ} \mathrm{C}, 190^{\circ} \mathrm{C}$, and $200{ }^{\circ} \mathrm{C}$ for $15 \mathrm{~min}$ using a coffee bean roaster (CBR-101; Gene Café, Ansan, Korea).

\subsection{Preparation of roasted ginseng powder}

Roasted ginseng roots were milled using SPEX 6875D Freezer/Mill (Spex SamplePrep, Metuchen, NJ, USA). The miller was precooled for $5 \mathrm{~min}$ to let it reach $-196{ }^{\circ} \mathrm{C}$, and then the sample was milled for $3 \mathrm{~min}$ at the maximum speed of 15 CPS (the number of back-and-forth cycles per second completed by the impactor). The miller was cooled for $1 \mathrm{~min}$ between each set of milling. 


\subsection{Treatments}

Grinding of the dried ginseng in different cryogenic milling conditions has been described in Fig. 1. Cryogenic milling condition was optimized by three steps, which included the milling cycle, milling speed (CPS), and time. Initial condition for the first cycle included precooling, 5 min; milling time, $3 \mathrm{~min}$; cooling time, $1 \mathrm{~min}$; and milling rate, 15 CPS. Range for each step was 2-12 for milling cycle, 5-15 CPS for milling rate, and 0.5-5 min for milling time. After cryogenic milling of ginseng samples under different conditions, the samples were classified based on their particle size. The particle size was determined on the value based on surface weighted meant, $\mathrm{D}[3,2]$, and the size ranges were 10-20 $\mu \mathrm{m}, 20-30 \mu \mathrm{m}, 30-40 \mu \mathrm{m}, 40-50 \mu \mathrm{m}$, and $>50 \mu \mathrm{m}$ (Table 2). One treatment condition for each size range was then selected, and samples selected based on particle size were roasted and pulverized by cryogenic milling. Pretreatment with roasting was performed at $160^{\circ} \mathrm{C}, 170^{\circ} \mathrm{C}, 180^{\circ} \mathrm{C}$, $190{ }^{\circ} \mathrm{C}$, and $200{ }^{\circ} \mathrm{C}$ for $15 \mathrm{~min}$ (Table 3).

a)

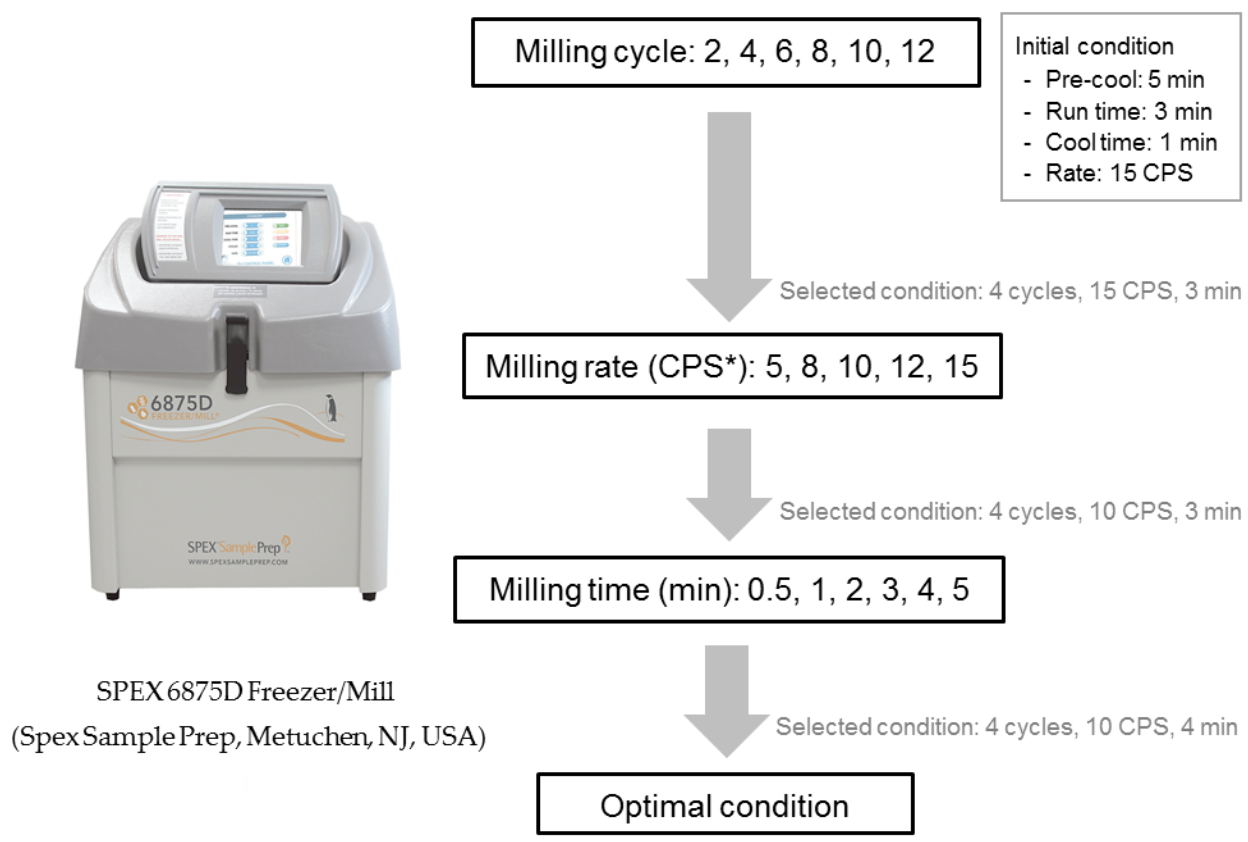

Fig. 1. Cryogenic miller (a) and cryogenic milling conditions (b) used for dried ginseng root. The conditions used for cycle- 1 were 3 min-milling at a rate of 15 CPS. CPS stands for the number of backand-forth cycles per second completed by the impactor.

\subsection{Particle size}

Particle size of the ginseng powder was measured based on following conditions: particle refractive index, 1.520; particle absorption index, 0.01; dispersant name, dry; dispersion media refractive index, 1; particle type, non-spherical; and measurement time, background $10 \mathrm{~s} /$ sample 10 s using particle size analyzer (Mastersizer 3000; Malvern, Worcestershire, UK). After placing the ginseng powder on the sample inlet, $50 \%$ of flow rate (power) and $50 \%$ of feed rate (power) were set to run. Average particle size of all ginseng powders was evaluated at least 5 times, and expressed by different designations.

Table 1. Particle size distribution measurement

\begin{tabular}{ll}
\hline Designation & Description \\
\hline $\mathrm{D}[3,2]$ & The surface area moment (Sauter) mean diameter \\
$\mathrm{D}[4,3]$ & The volume moment mean diameter \\
\hline
\end{tabular}


Dx $(10,50,90) \quad$ The cumulative percentiles

\subsection{Color}

Separate petri dishes $(35 \times 10 \mathrm{~mm})$ were completely covered with each type of ginseng powder. Color values of ginseng powder were measured using a colorimeter (CR-400; Konica Minolta Sensing, Tokyo, Japan) after calibrating with a standard $\left(L^{*}, 95.78 ; a^{*},-0.05\right.$; and $\left.b^{*}, 2.01\right)$. All ginseng powder samples were measured at least 5 times.

\subsection{Water solubility index (WSI)}

WSI of ginseng powder was measured in triplicates using the method described by Lee et al. (2013) [6]. Ginseng powder (0.5 g) was dispersed in $30 \mathrm{~mL}$ distilled water, and the solution was incubated at $80^{\circ} \mathrm{C}$ in a water bath shaker (BF-30SB; BioFree, Seoul, Korea) for $1 \mathrm{~h}$. Then, the solution was centrifuged at 10,000 rpm for 30 min using a centrifugal separator (1736R; GYROZEN, Daejeon, Korea). The remaining supernatant was filtered with Whatman No. 2 filter paper (Sigma-Aldrich, St. Louis, MO, USA) and the filtrate was dried at $60^{\circ} \mathrm{C}$ for $24 \mathrm{~h}$ using a Hot air dryer (LD-918TH; Lequip, Hawsung, Korea). WSI was calculated as the following equation:

WSI $(\%)=($ Weight of dried ginseng solution $/$ Weight of ginseng powder $) \times 100$

\subsection{Antioxidant extract preparation}

Each ginseng sample $(1 \mathrm{~g})$ was extracted with $70 \%$ ethanol $(25 \mathrm{~mL})$ for $3 \mathrm{~h}$ at $80{ }^{\circ} \mathrm{C}$ using a water bath (BF-30SB; Biofree), and ginseng extract was filtered with Whatman No. 2 filter paper (SigmaAldrich). Then, ginseng extract was concentrated using a rotary vacuum evaporator (EYELA rotary evaporator N-1000; Sunileyela, Seongnam, Korea), and freeze-dried using a freeze dryer (MCFD8512; Ilshin biobase, Yangju, Korea).

\subsubsection{DPPH free radical scavenging activity}

The scavenging activity of freeze-dried ginseng samples was measured using 2,2-diphenyl-1picryl-hydrazyl (DPPH) (Sigma, St. Louis, MO, USA) radical by modified Blois method (1958) [22]. Freeze-dried ginseng sample was diluted using HPLC grade methanol, and the ginseng solution (160 $\mu \mathrm{L})$ was mixed with $0.4 \mathrm{mM}$ DPPH $(40 \mu \mathrm{L}, \mathrm{Ab}$ 0.95-0.99). Similarly, to calibrate the color of the sample, methanol $(40 \mu \mathrm{L})$ instead of DPPH was added to the ginseng solution $(160 \mu \mathrm{L})$. Methanol $(160 \mu \mathrm{L})$ mixed with DPPH $(40 \mu \mathrm{L})$ was used as control. All reactions were performed in the dark at room temperature $\left(22-24^{\circ} \mathrm{C}\right)$ for $10 \mathrm{~min}$. Absorbance was measured at $517 \mathrm{~nm}$ by Multiskan ${ }^{\mathrm{TM}} \mathrm{GO}$ plate reader (Thermo Fisher Scientific, Waltham, MA, USA). DPPH free radical scavenging activity was determined by following equation, and the antioxidant activity was represented by $\mathrm{IC}_{50}$ value indicating the amount of sample that eliminated $50 \%$ of DPPH radical.

DPPH free radical scavenging activity $(\%)=[1-\{(\mathrm{A} 1-\mathrm{A} 2) / \mathrm{A} 3\}] \times 100$

A1: Absorbance of $40 \mu \mathrm{L}$ DPPH solution $+160 \mu \mathrm{L}$ ginseng solution

A2: Absorbance of $40 \mu \mathrm{L}$ methanol $+160 \mu \mathrm{L}$ ginseng solution

A3: Absorbance of $40 \mu \mathrm{L} \mathrm{DPPH}+160 \mu \mathrm{L}$ methanol

\subsection{Ginseng extract}

To obtain ginseng extract, ginseng powder $(0.5 \mathrm{~g})$ was diluted with distilled water $(50 \mathrm{~mL})$ at 95 ${ }^{\circ} \mathrm{C}$ for $2 \mathrm{~h}$ in a water bath (BF-30SB; Biofree). Further, ginseng solution was centrifuged at 10,000 rpm for 10 min using a centrifugal separator (1736R; Gyrozen, Daejeon, Korea), filtered, and final volume was adjusted to $50 \mathrm{~mL}$ volumetric flask for analyzing the total polyphenol content and the total and acidic polysaccharide contents. 


\subsubsection{Total polyphenol contents (TPC)}

TPC was analyzed by Folin-Denis method (Ough and Amerine 1980) [23]. Ginseng extract (2 $\mathrm{mL}$ ) and Folin-Ciocalteu reagent $(2 \mathrm{~mL}$ ) were mixed for 3 min followed by mixing $2 \mathrm{~mL}$ of $10 \%$ $\mathrm{Na}_{2} \mathrm{CO}_{3}$. The final mixture was reacted in dark at room temperature for $1 \mathrm{~h}$, and their absorbance was measured at $700 \mathrm{~nm}$ by a Multiskan ${ }^{\mathrm{TM}} \mathrm{GO}$ plate reader (Thermo Fisher Scientific). TPC of ginseng was evaluated by gallic acid (Sigma-Aldrich) standard curve $\left(y=15.111 x+0.0406, \mathrm{R}^{2}=0.9976\right)$.

\subsubsection{Total polysaccharide contents}

Total polysaccharide contents were measured by the phenol-sulfuric acid method as described by Dubois et al. 1956 [24]. Ginseng extract $(0.6 \mathrm{~mL})$ was mixed with $0.3 \mathrm{~mL}$ of $5 \%$ phenol and $1.5 \mathrm{~mL}$ of concentrated sulfuric acid. The mixture was incubated in a water bath shaker (BF-30SB; Biofree) at $85^{\circ} \mathrm{C}$ for $30 \mathrm{~min}$, and later, cooled for $5 \mathrm{~min}$ at room temperature in dark. Absorbance of final mixture $(0.2 \mathrm{~mL})$ was measured at $490 \mathrm{~nm}$ by a Multiskan ${ }^{\mathrm{TM}} \mathrm{GO}$ plate reader (Thermo Fisher Scientific). Total polysaccharide content of ginseng was evaluated by D-glucose (Samchun, Seoul, Korea) standard curve $\left(\mathrm{y}=0.4895 \mathrm{x}+0.0088, \mathrm{R}^{2}=0.9955\right)$.

\subsubsection{Acidic polysaccharide contents}

Acidic polysaccharide contents were measured by Carbazole-Sulfuric method as described by Do et al. 1993 [25]. Ginseng extract $(0.5 \mathrm{~mL})$ was mixed with $0.25 \mathrm{~mL}$ of $0.1 \%(\mathrm{v} / \mathrm{v})$ carbazole-absolute ethanol (Alfa Aesar, Haverhill, MA, USA) and $3 \mathrm{~mL} \mathrm{H}_{2} \mathrm{SO}_{4}$. The mixture was incubated in a water bath shaker (BF-30SB; Biofree) at $80^{\circ} \mathrm{C}$ for $5 \mathrm{~min}$, and cooled for $15 \mathrm{~min}$ at room temperature in the dark. Then, the absorbance of the final mixture $(0.2 \mathrm{~mL})$ was measured at $525 \mathrm{~nm}$. Acidic polysaccharide content of ginseng was evaluated by D-galacturonic acid (Sigma-Aldrich) standard curve $\left(y=3.5033 x+0.3208, R^{2}=0.9842\right)$.

\subsection{Ginsenoside content}

After adding ginseng powder $(2 \mathrm{~g})$ to $25 \mathrm{~mL}$ of $70 \%$ methanol, crude ginsenoside was extracted using a funnel shaker (RS-1; Jeio Tech, Dajeon, Korea) for $15 \mathrm{~min}$. Following extraction, remaining fluid was adjusted to $50 \mathrm{~mL}$ volumetric flask. Ginseng solution $(50 \mathrm{~mL})$ was centrifuged for $5 \mathrm{~min}$ at 3,500 rpm and the supernatant was filtered through $0.22 \mu \mathrm{m}$ membrane filter (Millipore, Burlington, MA, USA). Filtered sample was analyzed by HPLC (1260 Infinity II LC System; Agilent, Santa Clara, CA, USA) with a C18 column (Kinetex, $5 \mu \mathrm{m}, 250 \times 4.6 \mathrm{~mm}$; Phenomenex, Seoul, Korea). The column temperature was set up at $30{ }^{\circ} \mathrm{C}$. Gradient elution was established with water (solvent A) and acetonitrile (solvent $B$ ), and the flow velocity was maintained at $1.0 \mathrm{~mL} / \mathrm{min}$. HPLC gradient elution was set as follows: (0-5 min, A 80\%, B 20\%; $20 \mathrm{~min}$, A 77\%, B 23\%; $25 \mathrm{~min}, \mathrm{~A} 70 \%$, B 30\%; $45 \mathrm{~min}$, A $60 \%$, B 40\%; 55-65 min, A 50\%, B 50\%; and 70-75 min, A 80\%, B 20\%). Total run time of HPLC was 75 min. The injection amount was $10 \mu \mathrm{L}$ and the absorbance was determined at $203 \mathrm{~nm}$.

\subsection{Statistical analysis}

All analyses were performed in at least triplicates. Data was analyzed by duncan's multiple range test after one-way analysis of variance (ANOVA) using SPSS 22.0 software (SPSS Inc., USA). Differences were considered statistically significant at $p<0.05$.

\section{Results and Discussion}

\subsection{Particle size}

At first, in preliminary experiments, when wet milling and cryogenic milling were compared, wet milling showed optimal conditions at 1:10 (ginseng: distilled water) ratio, and particle size was found to be $114 \mu \mathrm{m}$ based on D [3,2] value (data not shown). In comparison, the particle size of dried ginseng using cryogenic milling was reported to be significantly reduced to $14 \mu \mathrm{m}$ (based on $\mathrm{D}[3,2]$ 
value). Therefore, cryogenic milling was considered effective as there was eight time reduction in particle size compared to that in wet milling used in the study. Cryogenic milling has been reported to increase the stiffness and decrease the flexibility of plant cell wall, effortlessly making the plant cell wall brittle, which further leads to rapid reduction in particle size [18]. As a result, in this study, ginseng powders of varying sizes from 10 to $50 \mu \mathrm{m}$ or higher were manufactured by different cycles, cycles per second (CPS), and running times using cryogenic milling equipment (Table 2). Further, 5 out of 15 treatments (treatment no. 1, 6, 11, 14, and 15 from Table 2) were selected based on size, and roasted as a pretreatment. Particle size of roasted powdered samples are shown in Table 3. Compared to control (not roasted), all roasted treatments showed significant reduction in size based on the D $[3,2]$ value (Table 3). We observed a decreasing trend in particle size with an increase in roasting temperature. A previous study reported that roasting weakened the starch-protein linkage and broke down the starch granules, ultimately reducing the particle size [26]. However, the 10-20 $\mu \mathrm{m}$ size powder generated using different treatments was roasted at $180^{\circ} \mathrm{C}$ while remaining powders were roasted at $190{ }^{\circ} \mathrm{C}$ or above; it was confirmed that the particle size increased again. Our results were consistent with that of a previous study that showed that flocculation occurs as particle size becomes smaller $[27,28]$. Such flocculation has been suggested to be caused by the van der Waals forces as surface energy increases with increase in surface area [29]. Though flocculation occurred, the flocculated particle size was smaller than that of the control. Therefore, pretreatment using roasting was concluded to be effective in reducing particle size.

Table 2. Treatment based on particle size of dried ginseng powder with various cryogenic milling conditions

\begin{tabular}{|c|c|c|c|c|c|c|c|c|c|}
\hline \multirow[b]{2}{*}{$\begin{array}{l}\text { Size } \\
(\mu \mathrm{m})\end{array}$} & \multirow[b]{2}{*}{ Treatment } & \multicolumn{3}{|c|}{$\begin{array}{l}\text { Treatment } \\
\text { conditions }\end{array}$} & \multicolumn{5}{|c|}{ Particle size $(\mu \mathrm{m})$} \\
\hline & & Cycle & CPS & Time & $\mathrm{D}[3,2]$ & $\mathrm{D}[4,3]$ & Dx (10) & Dx (50) & Dx (90) \\
\hline \multirow{3}{*}{$10-20$} & 1 & 4 & 10 & 4 & $14.05 \pm 0.21 \mathrm{jC}$ & $68.24 \pm 7.08^{\mathrm{iB}}$ & $5.78 \pm 0.15^{\mathrm{i}}$ & $28.49 \pm 0.93 \mathrm{gC}$ & $145.88 \pm 8.50^{\text {ів }}$ \\
\hline & 2 & 4 & 10 & 3 & $14.97 \pm 0.70^{\mathrm{B}}$ & $63.82 \pm 4.49^{\mathrm{iB}}$ & $6.13 \pm 0.31 \mathrm{jA}$ & $30.75 \pm 1.24 \mathrm{gB}^{\mathrm{B}}$ & $150.93 \pm 8.15^{\mathrm{iB}}$ \\
\hline & 3 & 4 & 10 & 5 & $15.52 \pm 0.61^{\mathrm{jA}}$ & $89.43 \pm 10.93^{\mathrm{hA}}$ & $6.20 \pm 0.16^{\mathrm{jA}^{\mathrm{A}}}$ & $32.15 \pm 1.74 \mathrm{gA}$ & $193.54 \pm 16.51^{\mathrm{hA}}$ \\
\hline \multirow{5}{*}{$20-30$} & 4 & 4 & 10 & 2 & $21.04 \pm 2.20^{\mathrm{iE}}$ & $114.43 \pm 5.47^{\mathrm{fgBC}}$ & $8.07 \pm 0.88^{\mathrm{iE}}$ & $47.55 \pm 1.42^{\mathrm{fC}}$ & $308.43 \pm 15.75^{\mathrm{fC}}$ \\
\hline & 5 & 4 & 8 & 3 & $22.54 \pm 0.43^{\mathrm{hD}}$ & $105.13 \pm 3.72 \mathrm{gC}$ & $8.91 \pm 0.26^{\mathrm{hD}}$ & $47.83 \pm 0.76^{\mathrm{fC}}$ & $263.75 \pm 11.08 \mathrm{gD}$ \\
\hline & 6 & 4 & 12 & 3 & $24.93 \pm 0.35 \mathrm{gC}$ & $113.71 \pm 4.23^{\mathrm{fgBC}}$ & $9.58 \pm 0.13 \mathrm{gc}$ & $57.15 \pm 0.77 \mathrm{eв}$ & $299.17 \pm 4.71^{\mathrm{fCD}}$ \\
\hline & 7 & 6 & 15 & 3 & $28.74 \pm 1.02^{\mathrm{eA}}$ & $131.47 \pm 13.30^{\mathrm{eA}}$ & $11.82 \pm 0.29$ ев & $53.69 \pm 2.79 \mathrm{eA}$ & $377.87 \pm 58.51^{\mathrm{dA}}$ \\
\hline & 8 & 4 & 15 & 3 & $29.57 \pm 0.64^{\mathrm{AB}}$ & $124.00 \pm 8.16^{\mathrm{fB}}$ & $13.78 \pm 0.15^{\mathrm{dA}}$ & $60.53 \pm 2.56^{\mathrm{eA}}$ & $345.50 \pm 40.43^{\mathrm{ев}}$ \\
\hline \multirow{5}{*}{$30-40$} & 9 & 8 & 15 & 3 & $31.67 \pm 1.16^{\mathrm{eC}}$ & $162.80 \pm 17.15^{\mathrm{eC}}$ & $12.92 \pm 0.31^{\mathrm{eB}}$ & $60.79 \pm 4.72^{\mathrm{eC}}$ & $522.33 \pm 54.00^{\mathrm{dC}}$ \\
\hline & 10 & 10 & 15 & 3 & $31.90 \pm 1.64 \mathrm{eC}$ & $177.00 \pm 14.38^{\mathrm{dB}}$ & $11.90 \pm 0.27 \mathrm{fD}$ & $61.05 \pm 4.34^{\mathrm{eC}}$ & $567.27 \pm 45.37 \mathrm{cB}$ \\
\hline & 11 & 4 & 10 & 1 & $34.48 \pm 0.82^{\mathrm{dB}}$ & $215.55 \pm 11.16^{\mathrm{bA}}$ & $12.44 \pm 0.28^{\mathrm{eC}}$ & $113.82 \pm 9.08^{\mathrm{bA}}$ & $591.73 \pm 24.49^{\mathrm{bcB}}$ \\
\hline & 12 & 12 & 15 & 3 & $40.02 \pm 2.77^{\mathrm{cA}}$ & $226.00 \pm 3.00^{\mathrm{bA}}$ & $15.20 \pm 0.54^{\mathrm{cA}}$ & $86.65 \pm 6.28^{\mathrm{dB}}$ & $664.20 \pm 33.04^{\mathrm{aA}}$ \\
\hline & 13 & 4 & 5 & 3 & $40.63 \pm 1.01^{\mathrm{cA}}$ & $217.63 \pm 6.44^{\mathrm{bA}}$ & $15.01 \pm 0.33^{\mathrm{cA}}$ & $111.25 \pm 4.43^{\mathrm{bA}}$ & $602.38 \pm 15.85^{\mathrm{bB}}$ \\
\hline $40-50$ & 14 & 2 & 15 & 3 & $46.83 \pm 0.99 \mathrm{~b}$ & $198.80 \pm 6.98^{c}$ & $19.03 \pm 0.38^{b}$ & $104.00 \pm 1.41^{c}$ & $529.40 \pm 22.83^{\mathrm{d}}$ \\
\hline$>50$ & 15 & 4 & 10 & 0.5 & $64.07 \pm 5.31^{\mathrm{a}}$ & $289.00 \pm 5.00^{\mathrm{a}}$ & $24.95 \pm 2.06^{\mathrm{a}}$ & $207.40 \pm 6.27^{a}$ & $690.40 \pm 10.60^{\mathrm{a}}$ \\
\hline
\end{tabular}

${ }^{a-j}$ Means with different superscripts within whole particle size group are significantly different $(p<0.05)$. ${ }^{\mathrm{A}-\mathrm{E}} \mathrm{Means}$ with different superscripts within similar size group are significantly different $(p<0.05)$. Particle size $(\mu \mathrm{m})$ established on the basis of D [3,2] value. Fifteen treatments were classified based on cycle, CPS (the number of back-and-forth cycles per second), and time conditions, and more number of treatments for larger particle size. One cycle is based on pulverization for 3 minutes at a rate of 15 CPS oscillating 15 times per second. 
Table 3. Particle size of dried ginseng powder at different roasting temperatures

\begin{tabular}{|c|c|c|c|c|c|c|}
\hline \multirow[b]{2}{*}{$\begin{array}{l}\text { Size } \\
(\mu \mathrm{m})\end{array}$} & \multicolumn{3}{|l|}{ Roasting1) } & \multicolumn{3}{|c|}{ article size $(\mu \mathrm{m})$} \\
\hline & $\begin{array}{l}\text { Temperature } \\
\left({ }^{\circ} \mathrm{C}\right)\end{array}$ & $\mathrm{D}[3,2]$ & $\mathrm{D}[4,3]$ & Dx (10) & Dx (50) & Dx (90) \\
\hline \multirow{6}{*}{$10-20$} & Control & $14.00^{\mathrm{eA}} \pm 0.20$ & $68.20^{\mathrm{eA}} \pm 7.10$ & $5.80^{\mathrm{eA}} \pm 0.20$ & $28.50^{\mathrm{eA}} \pm 0.90$ & $145.90^{\mathrm{eA}} \pm 8.50$ \\
\hline & 160 & $7.01^{\mathrm{dB}} \pm 0.24$ & $28.61^{\mathrm{dB}} \pm 1.69$ & $2.65^{\mathrm{d} C} \pm 0.14$ & $15.26^{\mathrm{dB}} \pm 0.97$ & $84.40^{\mathrm{dB}} \pm 4.15$ \\
\hline & 170 & $5.41^{\mathrm{eD} \pm 0.19}$ & $18.90^{\mathrm{e}} \pm 0.43$ & $2.17^{\mathrm{e}} \pm 0.04$ & $10.50^{\mathrm{e}} \pm 1.03$ & $50.36^{\mathrm{dD}} \pm 1.46$ \\
\hline & 180 & $5.60^{\mathrm{eCD}} \pm 0.12$ & $20.94^{\mathrm{dC}} \pm 0.88$ & $2.19 \mathrm{eDE}_{ \pm 0} 0.05$ & $10.29^{\mathrm{e}} \pm 0.63$ & $55.83^{\mathrm{eCD}} \pm 1.18$ \\
\hline & 190 & $5.78^{e} \mathrm{C}_{ \pm 0} .20$ & $20.93^{\mathrm{dC}} \pm 2.06$ & $2.33^{\mathrm{eD}} \pm 0.09$ & $10.64^{\mathrm{e} D} \pm 0.38$ & $57.94^{\mathrm{dC}} \pm 2.14$ \\
\hline & 200 & $6.99 \mathrm{e}^{\mathrm{B}} \pm 0.52$ & $23.93^{\mathrm{eBC}} \pm 1.55$ & $2.81^{\mathrm{eB}} \pm 0.16$ & $13.50^{\mathrm{eC}} \pm 2.15$ & $61.40^{\mathrm{e}} \pm 2.80$ \\
\hline \multirow{6}{*}{$20-30$} & Control & $24.90^{\mathrm{dA}} \pm 0.40$ & $113.70^{\mathrm{dA}} \pm 4.20$ & $9.60^{\mathrm{dA}} \pm 0.10$ & $57.20^{\mathrm{dA}} \pm 0.80$ & $299.20^{\mathrm{dA}} \pm 4.70$ \\
\hline & 160 & $12.98^{\mathrm{c}} \pm 0.35$ & $101.74^{\mathrm{c}} \pm 7.58$ & $5.29^{\mathrm{c} B} \pm 0.30$ & $39.64^{\mathrm{cdB}} \pm 2.34$ & $277.80^{\mathrm{cA}} \pm 22.60$ \\
\hline & 170 & $11.98^{\mathrm{dC}} \pm 0.33$ & $94.58^{\mathrm{dB}} \pm 15.83$ & $4.73^{\mathrm{d} C} \pm 0.07$ & $32.90^{\mathrm{d} C} \pm 1.27$ & $235.20^{\circ B} \pm 35.93$ \\
\hline & 180 & $9.80^{\mathrm{dE}} \pm 0.44$ & $52.40^{\mathrm{cD}} \pm 11.92$ & $3.87 \mathrm{dE} \pm 0.13$ & $22.56^{\mathrm{dE}} \pm 2.38$ & $118.35^{\mathrm{dD}} \pm 16.78$ \\
\hline & 190 & $10.68^{\mathrm{dD}} \pm 0.31$ & $50.18^{\mathrm{cD}} \pm 4.75$ & $4.36^{\mathrm{dD}} \pm 0.07$ & $25.08^{\mathrm{dD}} \pm 0.79$ & $136.67^{\mathrm{CD}} \pm 21.01$ \\
\hline & 200 & $13.33^{\mathrm{dB}_{ \pm} \pm 0.21}$ & $69.97 \mathrm{dC}_{ \pm} \pm .17$ & $5.30^{\mathrm{dB}} \pm 0.05$ & $33.45^{\mathrm{d} C_{ \pm}} \pm 0.68$ & $183.00 \mathrm{dC}_{ \pm} 8.12$ \\
\hline \multirow{6}{*}{$30-40$} & Control & $34.50^{\mathrm{cA}} \pm 0.80$ & $215.50^{\mathrm{bA}} \pm 11.20$ & $12.40^{\mathrm{cA}} \pm 0.30$ & $113.80^{\mathrm{bA}} \pm 9.10$ & $591.70^{\mathrm{bA}} \pm 24.50$ \\
\hline & 160 & $18.10^{\mathrm{bB}} \pm 0.38$ & $146.00^{\mathrm{bB}} \pm 6.16$ & $6.98^{\mathrm{bB}} \pm 0.38$ & $58.90^{\mathrm{bcB}} \pm 2.04$ & $431.20^{\mathrm{bB}} \pm 21.48$ \\
\hline & 170 & $17.43^{\mathrm{c}} \pm 0.42$ & $152.14^{\mathrm{cB}} \pm 14.24$ & $6.52^{\mathrm{c}} \pm 0.36$ & $61.80^{\mathrm{c} B} \pm 2.70$ & $439.00^{\mathrm{bB}} \pm 70.14$ \\
\hline & 180 & $12.66^{\mathrm{CE}} \pm 0.59$ & $62.40^{\mathrm{cD}} \pm 2.49$ & $4.92^{\mathrm{cE}} \pm 0.16$ & $31.21^{\mathrm{cD}} \pm 2.28$ & $180.80^{\mathrm{cD}} \pm 6.30$ \\
\hline & 190 & $13.96^{\mathrm{c} D \pm 0.58}$ & $88.04^{\mathrm{bC}} \pm 14.07$ & $5.51^{\mathrm{cD} \pm} \pm 0.30$ & $36.99 \mathrm{cCD}^{\mathrm{C}} \pm 1.97$ & $251.14^{\mathrm{bC}} \pm 42.44$ \\
\hline & 200 & $15.78^{\mathrm{c}} \pm 0.64$ & $101.02^{\mathrm{c}} \pm 12.28$ & $6.24^{\mathrm{c} C} \pm 0.33$ & $41.62^{\mathrm{c} C} \pm 2.72$ & $287.60^{c \mathrm{C}} \pm 39.18$ \\
\hline \multirow{6}{*}{$40-50$} & Control & $46.80^{\mathrm{bA}} \pm 1.00$ & $198.80^{\mathrm{cA}} \pm 7.00$ & $19.00^{\mathrm{bA}} \pm 0.40$ & $104.00^{\mathrm{cA}} \pm 1.40$ & $529.40^{\mathrm{CA}} \pm 22.80$ \\
\hline & 160 & $19.64^{\mathrm{bC} \pm 0.57}$ & $149.83^{\mathrm{bC} \pm} \pm 4.96$ & $7.71^{\mathrm{bC}} \pm 0.26$ & $68.20^{\mathrm{bC} \pm 3.16}$ & $439.00^{\mathrm{bB}} \pm 26.95$ \\
\hline & 170 & $21.76^{\mathrm{bB}} \pm 0.49$ & $164.43^{\mathrm{b}} \pm 2.88$ & $8.44^{\mathrm{bB} \pm 0.23}$ & $77.16^{\mathrm{bB} \pm 2.77}$ & $459.14^{\mathrm{bB}} \pm 6.72$ \\
\hline & 180 & $14.88^{\mathrm{bF}} \pm 0.53$ & $90.56^{\mathrm{bE} \pm 11.83}$ & $5.98^{\mathrm{bF} \pm 0.28}$ & $41.40^{\mathrm{bE}} \pm 0.88$ & $248.50^{\mathrm{bE}} \pm 29.22$ \\
\hline & 190 & $15.84^{\mathrm{bE}} \pm 0.23$ & $96.96^{\mathrm{bE}} \pm 8.11$ & $6.34^{\mathrm{bE} \pm 0.10}$ & $42.86^{\mathrm{bE}} \pm 1.18$ & $277.17^{\mathrm{b} D} \pm 18.78$ \\
\hline & 200 & $18.23^{\mathrm{bD}} \pm 0.50$ & $123.67^{\mathrm{b}} \pm 6.89$ & $7.09^{\mathrm{b} D} \pm 0.24$ & $53.93^{\mathrm{bD}} \pm 1.48$ & $350.00^{\mathrm{bC}} \pm 22.23$ \\
\hline \multirow{6}{*}{$>50$} & Control & $64.10^{\mathrm{aA}} \pm 5.30$ & $289.00^{a \mathrm{a}} \pm 5.00$ & $25.00^{\mathrm{aA}} \pm 2.10$ & $207.40^{\mathrm{aA}} \pm 6.30$ & $690.40^{\mathrm{aB}} \pm 10.60$ \\
\hline & 160 & $39.04^{\mathrm{aB}} \pm 4.57$ & $314.00^{\mathrm{aA}} \pm 31.87$ & $15.73^{\mathrm{a}} \pm 1.67$ & $239.19^{\mathrm{aA}} \pm 49.29$ & $739.40^{\mathrm{aA}} \pm 33.08$ \\
\hline & 170 & $28.29^{a C} \pm 1.62$ & $231.25^{\mathrm{aC}} \pm 12.87$ & $10.44^{\mathrm{aC}} \pm 0.43$ & $126.50^{\mathrm{a} \mathrm{B}} \pm 15.41$ & $645.25^{\mathrm{aC}} \pm 14.64$ \\
\hline & 180 & $25.58^{\mathrm{a}} \pm 0.10$ & $219.40^{\mathrm{a} C} \pm 4.28$ & $9.73^{a C_{ \pm}} \pm 0.12$ & $96.60^{\mathrm{aB}} \pm 3.32$ & $635.20^{\mathrm{a} C} \pm 10.28$ \\
\hline & 190 & $27.63^{\mathrm{a}} \pm 1.11$ & $234.75^{\mathrm{aC}} \pm 13.09$ & $10.43^{\mathrm{a}} \pm 0.28$ & $108.45^{\mathrm{a}} \pm 9.82$ & $642.88^{a} C_{ \pm} 23.12$ \\
\hline & 200 & $27.07^{\mathrm{aC}} \pm 1.13$ & $225.33^{\mathrm{a} C} \pm 13.99$ & $10.82^{\mathrm{aC}} \pm 0.47$ & $103.58^{\mathrm{aB}_{ \pm}} .7 .01$ & $640.88^{\mathrm{aC}} \pm 27.10$ \\
\hline
\end{tabular}

a-eMeans with different superscripts within same temperature group are significantly different $(p<0.05)$. ${ }^{\text {A-F Means }}$ with different superscripts within same size group are significantly different $(p<0.05)$. ${ }^{1)}$ After cryogenic milling of ginseng samples with different conditions, the samples were classified based on their particle sizes. One treatment condition was selected for each size range, and samples selected based on the particle size were roasted and pulverized by cryogenic milling.

\subsection{Appearance}

Fig. 2 depicts appearance of raw dried ginseng before and after roasting. As roasting temperature was increased, the color of ginseng roots was observed to change from brown to black. On roasting at temperatures above $190{ }^{\circ} \mathrm{C}$, we found that ginseng roots started appearing burnt. Following roasting, dried ginseng was ground using cryogenic mill. The powdered ginseng before and after roasting is shown in Fig. 3. As the particle size of the control group (before roasting) were increased, the powder appeared to be rougher and this was observed only with the control group. The roasted samples appeared to be softer than the control samples. We noticed that larger the particle size and higher the roasting temperature, darker was the color of the ginseng samples. 


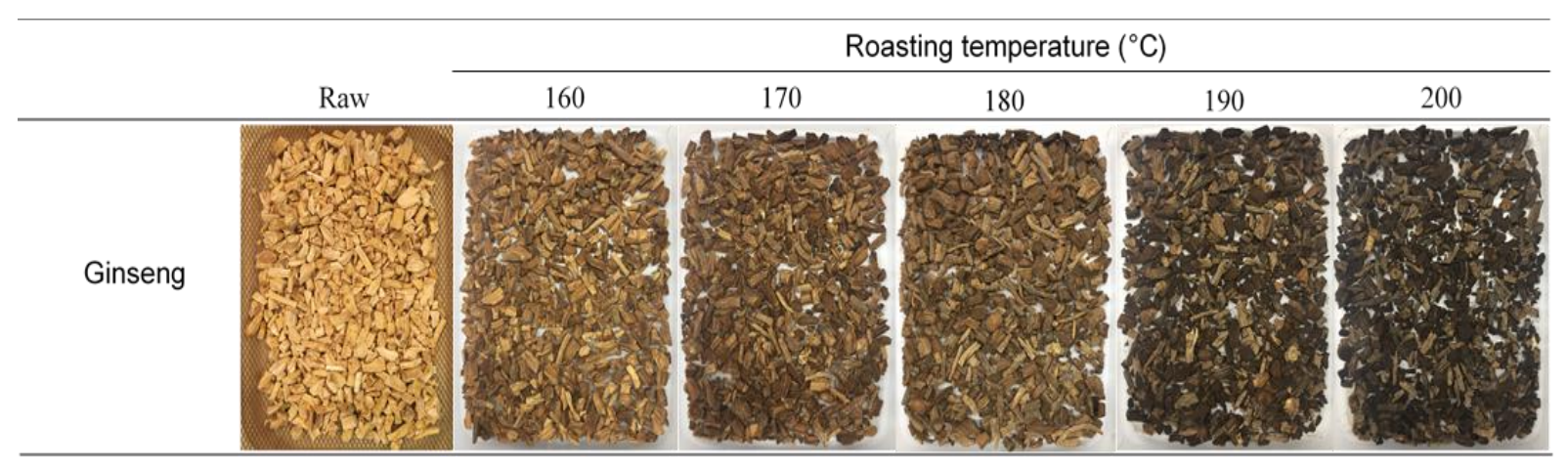

Figure 2. Dried ginseng root roasted at different temperatures

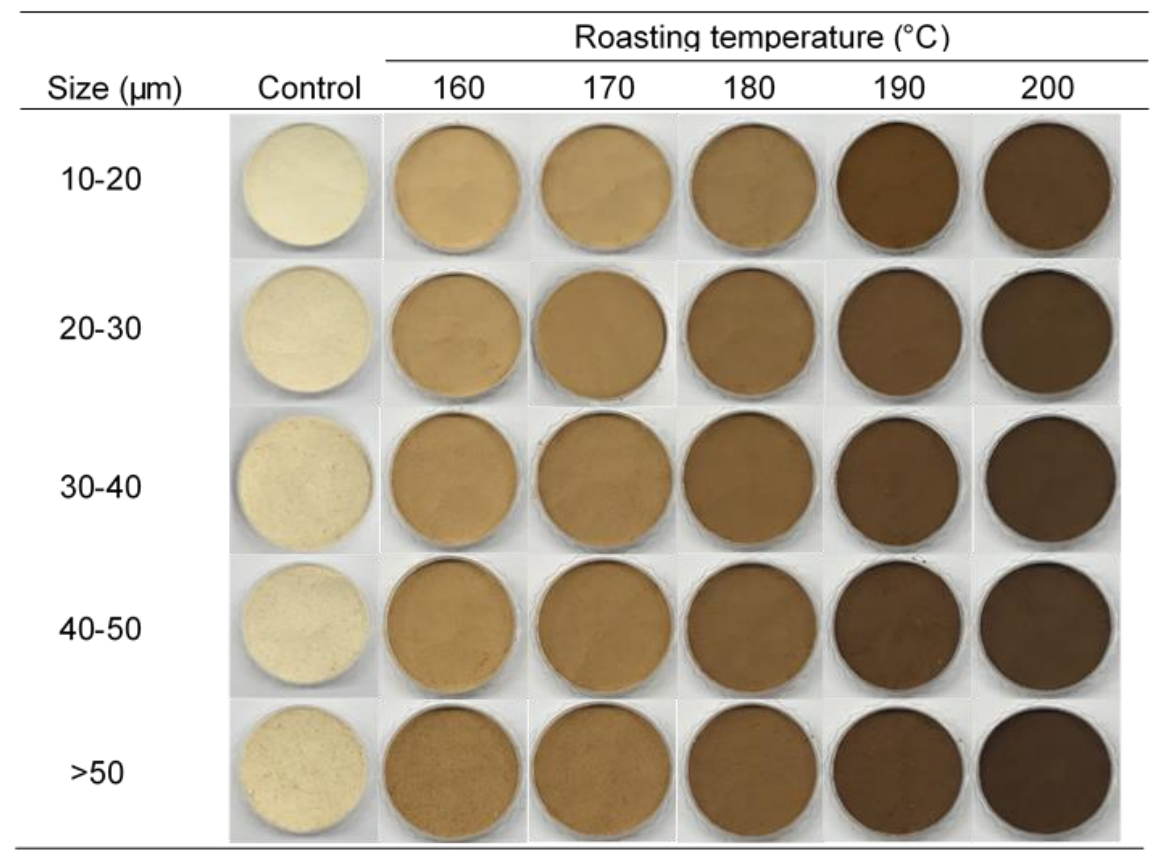

Figure 3. Dried ginseng root powders which were pretreated using different roasting temperatures

\subsection{Color}

Color values of ginseng powder are shown in Table 4. For control, we observed that smaller the particle size, higher the lightness $\left(\mathrm{L}^{*}\right)$ values and lower the redness $\left(\mathrm{a}^{*}\right)$ and yellowness $\left(\mathrm{b}^{*}\right)$ values. All values were significantly different from each other; the exception being $a^{*}$ and $b^{*}$ values of $50 \mu \mathrm{m}$ samples. For samples roasted at similar temperature, we found that smaller the particle size, higher the $\mathrm{L}^{*}$ value; lower $\mathrm{a}^{*}$ values up to $170{ }^{\circ} \mathrm{C}$ and higher $\mathrm{b}^{*}$ values were found. A previous study has reported that smaller sized ginseng powder $(600-1000 \mathrm{~nm})$ has higher $L^{*}$ value and lower $\mathrm{a}^{*}$ and $\mathrm{b}^{*}$ values compared to larger size ginseng powder $(300-500 \mu \mathrm{m})[19]$. Higher $L^{*}$ value has been shown to result from greater scattering of light by smaller particles [6].

Regarding the effect of roasting, $\mathrm{L}^{*}$ and $\mathrm{a}^{*}$ values of roasted samples revealed significantly lower and higher effect, respectively than the controls $(p<0.05)$, while no trend was observed on analyzing the $b^{*}$ values. On comparing same size range of samples that were roasted at different temperatures, we found that $L^{*}$ and $b^{*}$ values of all samples were significantly decreased with increase in the roasting temperature $(p<0.05)$. Furthermore, the $\mathrm{a}^{*}$ value of $>30 \mu \mathrm{m}$-sized samples was found to be significantly decreased with increase in roasting temperature, while for 10-30 $\mu \mathrm{m}$-sized samples, $\mathrm{a}^{*}$ value was observed to increase up to $170{ }^{\circ} \mathrm{C}$ thereafter, decreased up to $200{ }^{\circ} \mathrm{C}$. Seong et al. (2018) reported that such color change was due to generation of hydroxymethylfurfural (HMF) and pyrazine, the browning materials produced at high roasting temperature [30]. Consistent with our 
results, Park et al. (1995) reported that when ginseng marc powder is pretreated by roasting, the $L^{*}$ and $b^{*}$ values decrease with an increase in roasting temperature, while $a^{*}$ value increases up to 170 ${ }^{\circ} \mathrm{C}$ and decreases up to $230{ }^{\circ} \mathrm{C}$ [31]. This confirmed that the color parameters of ginseng powder were affected by particle size as well as roasting temperature.

Table 4. Color value of dried ginseng powder at different roasting temperatures

\begin{tabular}{|c|c|c|c|c|c|c|c|c|c|c|}
\hline \multirow[b]{2}{*}{ Size $(\mu \mathrm{m})$} & \multirow{2}{*}{$\begin{array}{c}\text { Roasting }{ }^{11} \\
\text { Temperature }\left({ }^{\circ} \mathrm{C}\right)\end{array}$} & \multicolumn{7}{|c|}{ Color } & \multirow{2}{*}{\multicolumn{2}{|c|}{$b^{*}$}} \\
\hline & & & $\mathrm{L}^{*}$ & & & $\mathrm{a}^{*}$ & & & & \\
\hline \multirow{6}{*}{$10-20$} & Control & $82.13^{\mathrm{aA}}$ & \pm & 0.54 & $0.71^{\mathrm{dC}}$ & \pm & 0.22 & $15.40^{\mathrm{dC}}$ & \pm & 0.14 \\
\hline & 160 & $65.00^{\mathrm{aB}}$ & \pm & 0.24 & $5.44^{\mathrm{eA}}$ & \pm & 0.10 & $17.62^{\mathrm{aA}}$ & \pm & 0.11 \\
\hline & 170 & $59.77^{\mathrm{ac}}$ & \pm & 0.16 & $5.47^{\mathrm{eA}}$ & \pm & 0.02 & $16.50^{\mathrm{aB}}$ & \pm & 0.03 \\
\hline & 180 & $52.35^{\mathrm{aD}}$ & \pm & 0.08 & $5.44^{\mathrm{dA}}$ & \pm & 0.03 & $14.32^{\mathrm{aD}}$ & \pm & 0.06 \\
\hline & 190 & $43.29^{a E}$ & \pm & 0.12 & $5.43^{\mathrm{aA}}$ & \pm & 0.03 & $11.45^{\mathrm{aE}}$ & \pm & 0.01 \\
\hline & 200 & $37.04^{\mathrm{aF}}$ & \pm & 0.08 & $4.21^{\mathrm{aB}}$ & \pm & 0.01 & $7.79^{\mathrm{aF}}$ & \pm & 0.02 \\
\hline \multirow{6}{*}{$20-30$} & Control & $80.44^{\mathrm{bA}}$ & \pm & 0.25 & $1.07^{\mathrm{cF}}$ & \pm & 0.04 & $16.03^{\mathrm{cB}}$ & \pm & 0.36 \\
\hline & 160 & $60.47^{\mathrm{bB}}$ & \pm & 0.08 & $6.35^{\mathrm{dB}}$ & \pm & 0.03 & $17.29^{\mathrm{bA}}$ & \pm & 0.03 \\
\hline & 170 & $54.46^{\mathrm{bC}}$ & \pm & 0.08 & $6.49^{\mathrm{cA}}$ & \pm & 0.01 & $15.87^{\mathrm{bB}}$ & \pm & 0.07 \\
\hline & 180 & $44.59 \mathrm{bD}$ & \pm & 0.09 & $6.04^{\mathrm{bC}}$ & \pm & 0.05 & $11.90^{\mathrm{c} C}$ & \pm & 0.03 \\
\hline & 190 & $39.71^{\mathrm{bE}}$ & \pm & 0.08 & $5.18^{\mathrm{bD}}$ & \pm & 0.04 & $9.29 \mathrm{bD}$ & \pm & 0.01 \\
\hline & 200 & $35.12^{\mathrm{bF}}$ & \pm & 0.12 & $4.01^{\mathrm{bE}}$ & \pm & 0.01 & $6.52^{\mathrm{bE}}$ & \pm & 0.06 \\
\hline \multirow{6}{*}{$30-40$} & Control & $78.77^{\mathrm{cA}}$ & \pm & 0.77 & $1.41^{\mathrm{bF}}$ & \pm & 0.09 & $16.64^{\mathrm{bB}}$ & \pm & 0.26 \\
\hline & 160 & $57.47 \mathrm{cв}$ & \pm & 0.12 & $6.78^{\mathrm{cA}}$ & \pm & 0.07 & $16.96^{\mathrm{cA}}$ & \pm & 0.22 \\
\hline & 170 & $53.37 \mathrm{cc}$ & \pm & 0.05 & $6.45^{\mathrm{dB}}$ & \pm & 0.02 & $15.34^{\mathrm{c} C}$ & \pm & 0.04 \\
\hline & 180 & $43.83^{\mathrm{CD}}$ & \pm & 0.16 & $6.32^{\mathrm{aC}}$ & \pm & 0.02 & $12.28^{\mathrm{bD}}$ & \pm & 0.02 \\
\hline & 190 & $37.72^{\mathrm{cE}}$ & \pm & 0.15 & $4.82^{\mathrm{cD}}$ & \pm & 0.01 & $8.38^{\mathrm{cE}}$ & \pm & 0.00 \\
\hline & 200 & $34.38^{\mathrm{cF}}$ & \pm & 0.12 & $3.84^{\mathrm{cE}}$ & \pm & 0.01 & $6.28^{\mathrm{cF}}$ & \pm & 0.02 \\
\hline \multirow{6}{*}{$40-50$} & Control & $77.57^{\mathrm{dA}}$ & \pm & 0.04 & $1.83^{\mathrm{aF}}$ & \pm & 0.02 & $17.21^{\mathrm{aA}}$ & \pm & 0.01 \\
\hline & 160 & $56.05^{\mathrm{dB}}$ & \pm & 0.10 & $6.91^{\mathrm{bA}}$ & \pm & 0.01 & $16.74^{\mathrm{dB}}$ & \pm & 0.07 \\
\hline & 170 & $50.72^{\mathrm{dC}}$ & \pm & 0.09 & $6.80^{\mathrm{bB}}$ & \pm & 0.02 & $14.87 \mathrm{dC}$ & \pm & 0.02 \\
\hline & 180 & $43.36^{\mathrm{dD}}$ & \pm & 0.03 & $5.91^{\mathrm{cC}}$ & \pm & 0.03 & $11.23^{\mathrm{dD}}$ & \pm & 0.05 \\
\hline & 190 & $37.47 \mathrm{dE}$ & \pm & 0.09 & $4.69 \mathrm{dD}$ & \pm & 0.05 & $8.06^{\mathrm{dE}}$ & \pm & 0.07 \\
\hline & 200 & $33.17 \mathrm{dF}$ & \pm & 0.08 & $3.35^{\mathrm{dE}}$ & \pm & 0.02 & $5.27 \mathrm{dF}$ & \pm & 0.03 \\
\hline \multirow{6}{*}{$>50$} & Control & $76.31^{\mathrm{eA}}$ & \pm & 0.10 & $1.91^{\mathrm{aF}}$ & \pm & 0.05 & $17.26^{\mathrm{aA}}$ & \pm & 0.01 \\
\hline & 160 & $52.12^{\mathrm{eB}}$ & \pm & 0.07 & $7.40^{\mathrm{aA}}$ & \pm & 0.03 & $15.86^{\mathrm{eB}}$ & \pm & 0.05 \\
\hline & 170 & $49.69^{\mathrm{eC}}$ & \pm & 0.14 & $7.14^{\mathrm{aB}}$ & \pm & 0.02 & $14.83^{\mathrm{dC}}$ & \pm & 0.01 \\
\hline & 180 & $41.77^{\mathrm{eD}}$ & \pm & 0.06 & $5.95^{\mathrm{cC}}$ & \pm & 0.04 & $10.84^{\mathrm{eD}}$ & \pm & 0.03 \\
\hline & 190 & $35.63^{\mathrm{eE}}$ & \pm & 0.08 & $4.50^{\mathrm{eD}}$ & \pm & 0.02 & $7.13^{\mathrm{eE}}$ & \pm & 0.05 \\
\hline & 200 & $33.19 \mathrm{dF}$ & \pm & 0.12 & $2.97 \mathrm{eE}$ & \pm & 0.02 & $4.75^{\mathrm{eF}}$ & \pm & 0.02 \\
\hline
\end{tabular}

a-e Means with different superscripts within same temperature group are significantly different $(p<0.05) .{ }^{\text {A-F Means }}$ with different superscripts within same size group are significantly different $(p<0.05) .{ }^{1)}$ After cryogenic milling of ginseng samples in different conditions, samples were classified based on their particle sizes. One treatment condition was selected for each size range, and particle size-based selected samples were roasted and pulverized by cryogenic milling.

\subsection{WSI}

WSI value of ginseng powders of different particle size and pretreated at different roasting temperatures were analyzed (Fig. 4). Significant change in WSI values was observed between samples in the size range of $10-20 \mu \mathrm{m}$ and $>50 \mu \mathrm{m}$; the exception being samples roasted at $170{ }^{\circ} \mathrm{C}$. WSI values 
of powdered samples of 10-20 $\mu \mathrm{m}$ size were found to be higher compared to those of larger sized samples, and WSI of powdered samples $(10-20 \mu \mathrm{m})$ roasted at $160{ }^{\circ} \mathrm{C}$ was found to be significantly higher than those with other treatments $(p<0.05)$. These results can be explained by particle sizesolubility relationship [18,32]. Not only particle size, but also roasting pretreatment was found to have significant effect on WSI. Furthermore, WSI values of all treatments were found to be higher than those of control samples, except for samples roasted at $190{ }^{\circ} \mathrm{C}$ and $200{ }^{\circ} \mathrm{C}$. This might be due to dextrinization, which can physically tears apart the starch granule at high temperature [33]. Additionally, it might be caused by breaking down of the amylopectin chain (insoluble molecules) into amylose (soluble molecules), with the increase in the amount of soluble polysaccharides [33]. Furthermore, Zavareze and Dias (2011) have reported that glycosidic bonds degrade at high temperature (approximately $80{ }^{\circ} \mathrm{C}$ ) leading to exposure of glucose (soluble dietary fiber) and increased WSI value [34]. However, in our study, when pretreatment with roasting was done at temperatures above $190^{\circ} \mathrm{C}$, WSI values were found to be decreased. The phenomenon was consistent with previous study that the amount of water-soluble compounds starts to decrease at $200{ }^{\circ} \mathrm{C}$ after $10 \mathrm{~min}$, and thereby, decreasing WSI [31]. Our results can be related to a burning phenomenon which is observed above $190{ }^{\circ} \mathrm{C}$ (Fig. 2). High roasting temperature may result in browning reaction (Maillard), which may produce high-molecular weight insoluble polymers from soluble lowmolecular weight polymers [35].

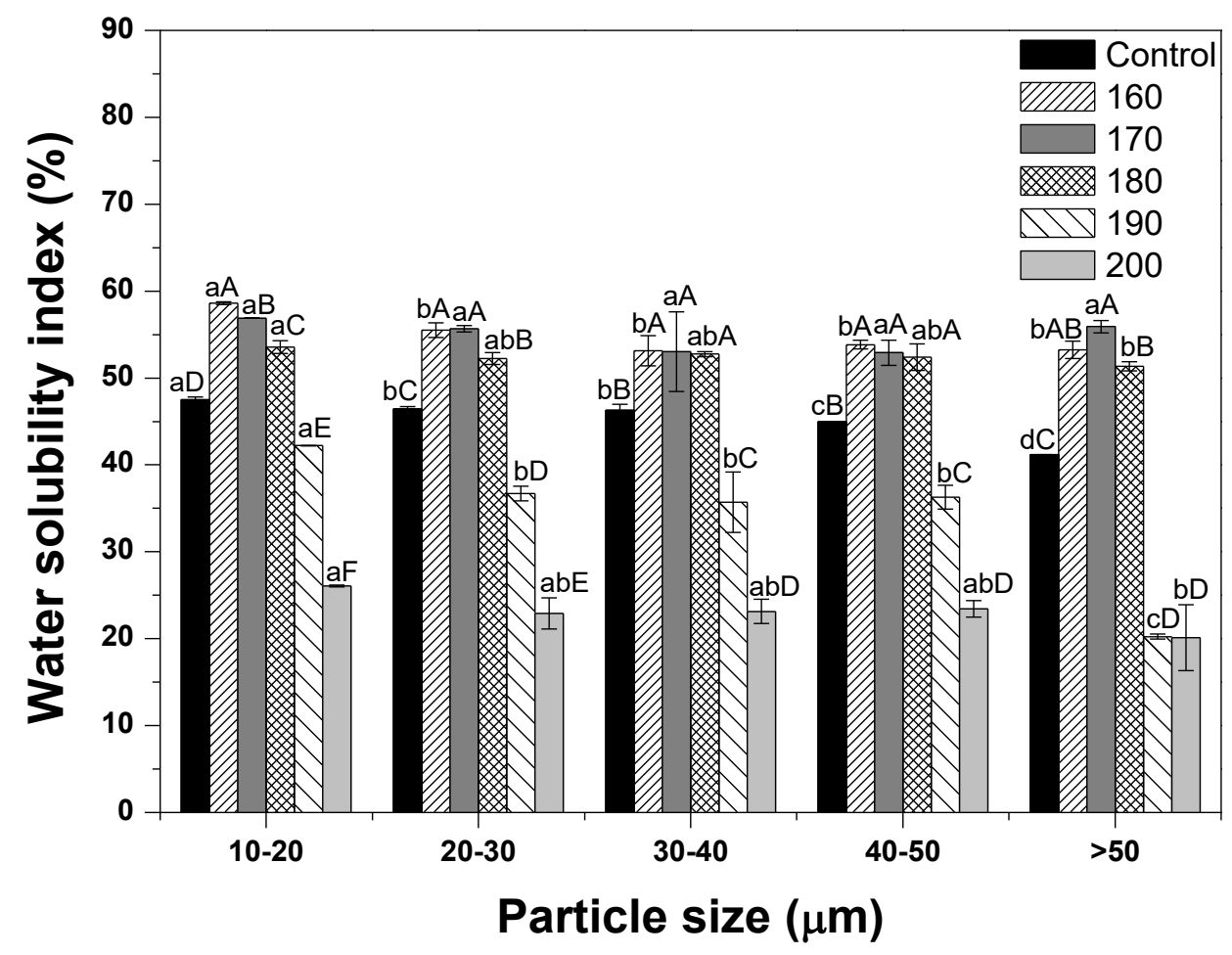

Figure 4. The water solubility index of dried ginseng powders with different particle sizes according to roasting temperature. After cryogenic milling of ginseng samples using different conditions, samples were classified based on their particle sizes. One treatment condition was selected for each size range, and the samples based on particle sizes were roasted and pulverized by cryogenic milling. ${ }^{\mathrm{a}-\mathrm{d}}$ Means with different superscripts within same temperature group are significantly different $(p<0.05)$. ${ }^{\text {A-F } M e a n s ~ w i t h ~ d i f f e r e n t ~}$ superscripts within same size group are significantly different $(p<0.05)$.

\subsection{Antioxidant activity}

Fig. 5 shows the antioxidant activity of ginseng powders which were pretreated using different roasting temperatures. With decrease in particle size, an increase in the antioxidant activity was 
observed, with the exception of samples roasted at $190{ }^{\circ} \mathrm{C}$ and above. All roasted powdered samples showed significantly lower 2,2-diphenyl-1-picrylhydrazyl (DPPH) radical scavenging activity (higher antioxidant activities) than the control samples. Ginseng powder samples of 10-20 $\mu \mathrm{m}$ showed the highest antioxidant activity compared to larger sized samples, except for samples roasted above $190{ }^{\circ} \mathrm{C}(p<0.05)$. On comparing the roasting temperatures, an increase in antioxidant activities was observed for each size group.

Pretreatment with roasting was observed to produce Maillard reaction products (melanoidin) due to thermal reaction between reducing sugars and nitrogen oxides, and an increase in antioxidant activity [17]. Our results were consistent with those of a previous study, which reported that antioxidant activity increases as roasting temperature increases from $130{ }^{\circ} \mathrm{C}$ to $170{ }^{\circ} \mathrm{C}$ [30].

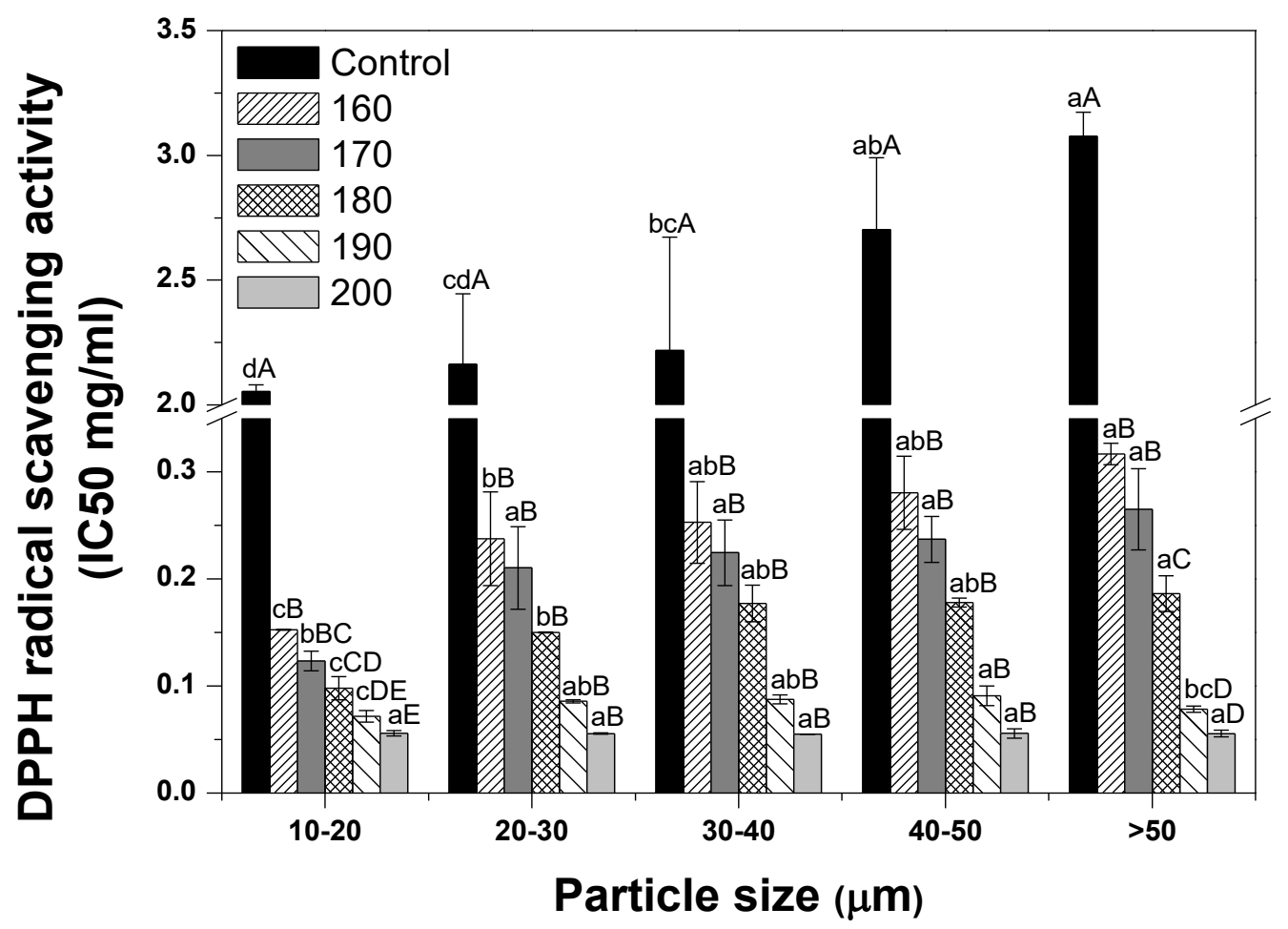

Figure 5. DPPH radical scavenging activity of dried ginseng powders with different particle sizes according to roasting temperature. After cryogenic milling of ginseng samples in different conditions, samples were classified based on their particle sizes. One treatment condition was selected for each size range, and samples selected based on particle size were roasted and pulverized by cryogenic milling. ${ }^{a-d}$ Means with different superscripts within same temperature group are significantly different $(p<0.05)$. A-EMeans with different superscripts within same size group are significantly different $(p<0.05)$.

\subsection{TPC}

TPC values of ginseng powder are shown in Fig. 6. We found a slight increase in TPC values with decrease in particle size $(p>0.05)$. All roasted treatments showed significantly higher TPC values than the control $(p<0.05)$. Thermal process converts the bioactive components like phenolic acid into their free forms. It has been known that elution from the food matrix is higher due to collapse of the cell membrane and cell wall [36]. Previous studies have reported that TPC is increased by intermediate substances such as reductone, which are produced by the browning reaction [36,37]. Reductone contains stable endiol structure that functions as an antioxidant by degrading the free radical chain, which results in generation of free hydrogen atoms $[37,38]$. Other studies have also reported that the bound phenolic compounds become freely available on heat treatment, and that 
high molecular phenolic compounds get transformed into low molecular compounds, thereby facilitating the extraction of polyphenol constituent $[39,40]$. Overall, we observed that TPC values of all ginseng samples increased with increase in roasting temperature below $180^{\circ} \mathrm{C}$.

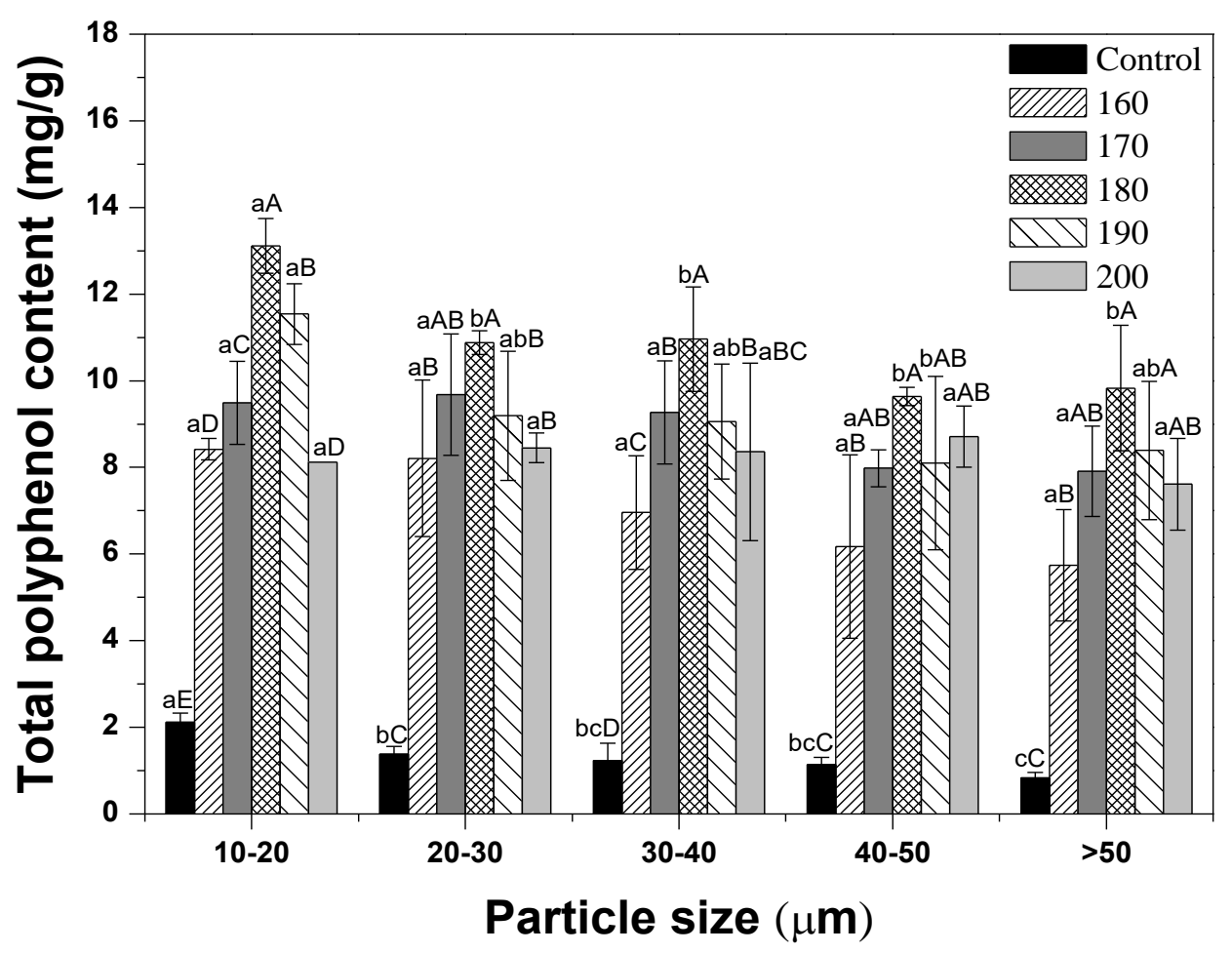

Figure 6. Total polyphenol content of dried ginseng powders with different particle sizes according to roasting temperature. After cryogenic milling of ginseng samples in different conditions, samples were classified based on their particle sizes. One treatment condition was selected for each size range, and samples selected based on particle size were roasted and pulverized by cryogenic milling. ${ }^{a-}$ cMeans with different superscripts within same temperature group are significantly different $(p<0.05)$. A-EMeans with different superscripts within same size group are significantly different $(p<0.05)$.

\subsection{Polysaccharide content}

Polysaccharides are bioactive components of the non-saponin system present in ginseng. These have bioactive functions, such as antioxidant activity, immune enhancement, and anti-fatigue [4143]. The total and acidic polysaccharide contents of ginseng powder samples were analyzed (Fig. 7a and $7 \mathrm{~b}$ ). The total polysaccharide content of $10-20 \mu \mathrm{m}$-size samples was significantly increased compared to that of $>50 \mu \mathrm{m}$-size samples $(p<0.05)$. Moreover, the content of acidic polysaccharides showed a similar pattern with the exception of control samples $(p<0.05)$. Cho et al. (2010) showed that less than $150 \mu \mathrm{m}$-size ginseng samples had higher total saccharide yields than larger sized samples [44]. According to roasting temperature, total and acidic polysaccharide contents were found to increase up to $180^{\circ} \mathrm{C}$, and this result might be due to increased solubilization of polysaccharide at high temperature [45]. Increase in the total polysaccharide content might be caused due to dramatic increase in acidic polysaccharide contents. It is thought that roasting decomposed the starch components, which promoted extraction of acidic polysaccharides [46]. However, total and acidic polysaccharide contents were found to be significantly decreased at $190^{\circ} \mathrm{C}$ and above $(p<0.05)$, which might be due to increase in inner constituents of ginger when burnt at high temperature. The levels of total polysaccharides were lower than control when roasted at $190{ }^{\circ} \mathrm{C}$ and above, while acidic polysaccharides were higher than control. Zhang et al. (2009) have shown that there are acidic as well as neutral type of polysaccharides in ginseng. It is hypothesized that neutral polysaccharides are 
mainly degraded at high temperature, resulting in a decrease in their levels. As acidic polysaccharides are known to be responsible for most of the biological activities in ginseng [47], roasting was considered to be an effective pretreatment.
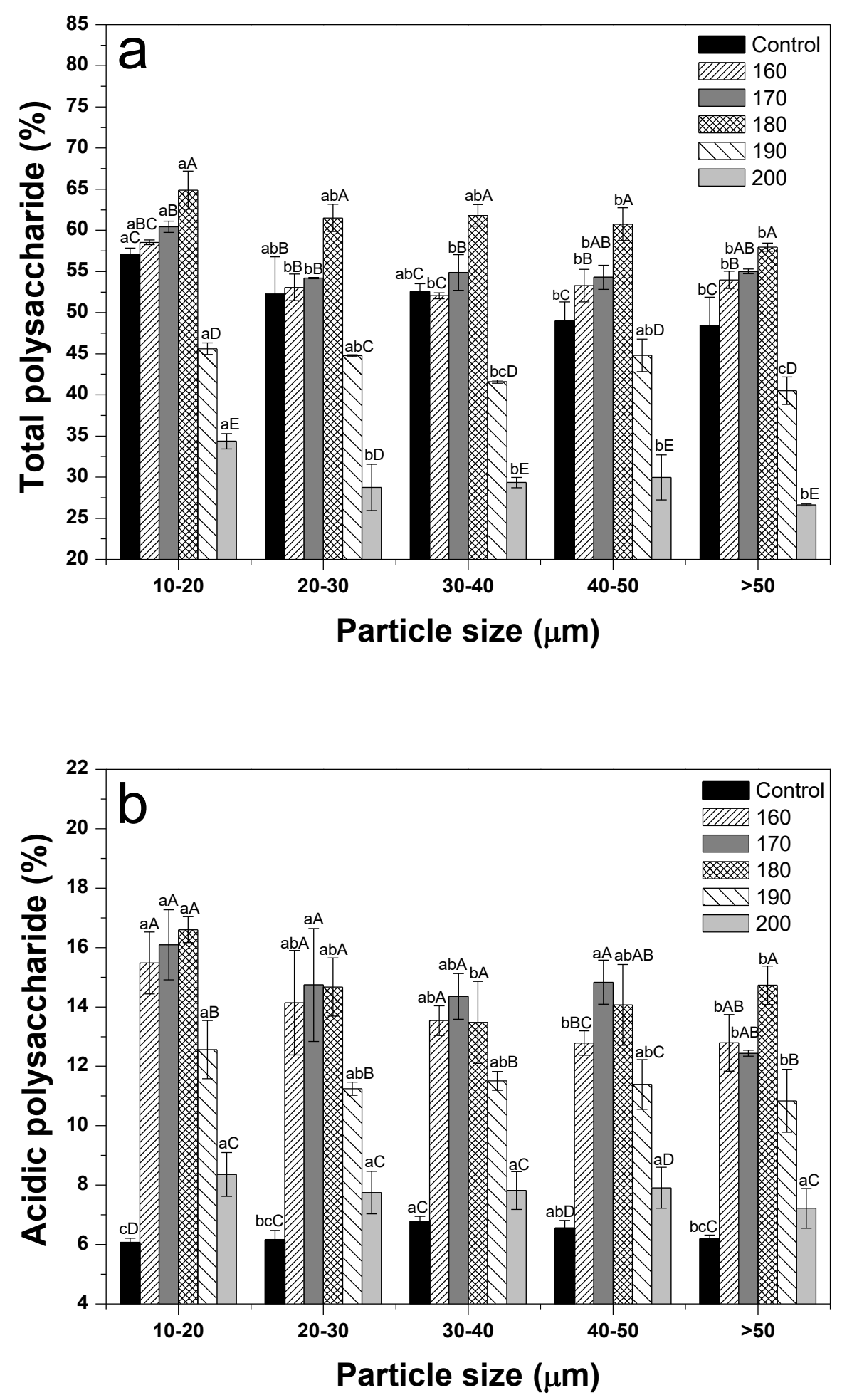

Figure 7. Total polysaccharide content (a) and acidic polysaccharide content (b) of dried ginseng powders with different particle size according to roasting conditions. After cryogenic milling of ginseng samples in different conditions, samples were classified based on their particle sizes. One treatment condition was selected for each size range, and particle size-based selected samples were roasted and pulverized by cryogenic milling. ${ }^{a-c}$ Means with different superscripts within same 
temperature group are significantly different $(p<0.05)$. A-EMeans with different superscripts within same size group are significantly different $(p<0.05)$.

\subsection{Ginsenoside contents}

The ginsenoside content of all ginseng samples did not show any alteration based on particle size (Table 5). However, dramatic differences were observed according to roasting temperature. Consistent with the findings of previous studies, we found that the amount of polar ginsenosides (Rg1, Re, Rf, Rb1, Rc, Rb2, Rb3, and Rd) decreased while that of non-polar ginsenosides [Rg2(S), $\operatorname{Rg} 2(\mathrm{R}), \operatorname{Rg} 3(\mathrm{~S}), \operatorname{Rg} 3(\mathrm{R})$, and $\mathrm{Rh} 1(\mathrm{~S})]$ increased as the roasting temperature was increased $[48,49]$. Most of the genosides, $\mathrm{Rb} 1, \mathrm{Re}$, and $\mathrm{Rg} 1$ which are known as main ingredients of ginseng tended to decrease with increase in roasting temperature. Such a trend was caused by degradation of the glycosidic bonds in the inner saponin structure at $170^{\circ} \mathrm{C}$ and above [30]. Roasting has been reported to increase $\mathrm{Rg} 2, \mathrm{Rg} 3$, and Rh1 levels due to hydrolysis reaction upon heat treatment [50]. Furthermore, roasting has been shown to convert Re into $\operatorname{Rg} 2$ ( $S$ and $R$ ) and Rh1, which is caused due to the chemical transformations such as epimerization and hydroxylation [50]. A previous study has reported that $\mathrm{Rg} 3$, a representative physiological active substance of ginseng, tends to increase on heating due to the weakening of the binding interaction among saponin structures [30]. Total ginsenoside contents were found to gradually decrease as roasting temperature was increased. Specifically, a dramatic decrease in ginsenoside contents was observed at $190{ }^{\circ} \mathrm{C}$ and above. Therefore, we concluded that pretreatment using roasting at too high temperature should not be considered as having a positive effect.

Table 5. Ginsenoside contents of dried ginseng powder at different roasting temperatures

\begin{tabular}{|c|c|c|c|c|c|c|c|c|c|}
\hline \multirow[b]{2}{*}{$\begin{array}{l}\text { Size } \\
(\mu \mathrm{m})\end{array}$} & \multicolumn{4}{|l|}{ Roasting1) } & \multicolumn{5}{|c|}{ Ginsenosides content (mg/g) } \\
\hline & $\begin{array}{l}\text { Temperature } \\
\left({ }^{\circ} \mathrm{C}\right)\end{array}$ & $\operatorname{Rg} 1$ & $\operatorname{Re}$ & $\mathrm{Rf}$ & $\mathrm{Rb} 1$ & Rc & $\mathrm{Rb} 2$ & $\mathrm{Rb} 3$ & $\mathrm{Rd}$ \\
\hline \multirow{13}{*}{$10-20$} & Control & $3.68^{\mathrm{A}} \pm 0.03$ & $3.41^{\mathrm{A}} \pm 0.02$ & $1.46^{\mathrm{A}} \pm 0.08$ & $4.26^{\mathrm{A}} \pm 0.03$ & $1.14^{\mathrm{B}} \pm 0.04$ & $0.97^{\mathrm{A}} \pm 0.03$ & $0.19 \mathrm{AB}_{ \pm 0.02}$ & $0.31^{\mathrm{A}} \pm 0.01$ \\
\hline & 160 & $3.72^{\mathrm{A}} \pm 0.08$ & $2.37^{\mathrm{B}} \pm 0.04$ & $0.86^{\mathrm{C}} \pm 0.00$ & $3.80^{\mathrm{B}} \pm 0.08$ & $1.32^{\mathrm{A}} \pm 0.05$ & $1.07^{\mathrm{A}} \pm 0.01$ & $0.23^{\mathrm{A}} \pm 0.06$ & $0.21^{\mathrm{B}} \pm 0.02$ \\
\hline & 170 & $3.41^{\mathrm{B}} \pm 0.06$ & $2.45^{\mathrm{B}} \pm 0.04$ & $0.93^{\mathrm{B}} \pm 0.01$ & $4.30^{\mathrm{A}} \pm 0.14$ & $1.37^{\mathrm{A}} \pm 0.05$ & $1.04^{\mathrm{A}} \pm 0.02$ & $0.23^{\mathrm{A}} \pm 0.06$ & $0.29 \mathrm{~A}^{\mathrm{A}} \pm 0.01$ \\
\hline & 180 & $2.30^{\mathrm{C}} \pm 0.14$ & $1.84^{\mathrm{C}} \pm 0.10$ & $0.73^{\mathrm{D}} \pm 0.01$ & $3.04^{C} \pm 0.03$ & $1.05^{\mathrm{C}} \pm 0.03$ & $0.92^{\mathrm{A}} \pm 0.01$ & $0.17^{\mathrm{AB}_{ \pm}}{ }_{0.01}$ & $0.18^{\mathrm{C}} \pm 0.01$ \\
\hline & 190 & $0.70^{\mathrm{D}} \pm 0.02$ & $0.93^{\mathrm{D}} \pm 0.02$ & $0.56^{\mathrm{E}} \pm 0.01$ & $1.32^{\mathrm{D}} \pm 0.01$ & $0.55^{\mathrm{D}} \pm 0.07$ & $0.50^{\mathrm{B}} \pm 0.03$ & $0.12^{\mathrm{B}} \pm 0.08$ & $0.11^{\mathrm{D}} \pm 0.03$ \\
\hline & 200 & $0.11^{\mathrm{E}} \pm 0.02$ & $0.18^{\mathrm{E}} \pm 0.01$ & $0.26^{\mathrm{F}} \pm 0.01$ & $0.26^{\mathrm{E}} \pm 0.01$ & $0.12^{\mathrm{E}} \pm 0.04$ & $0.39^{\mathrm{B}} \pm 0.46$ & $0.00^{C} \pm 0.00$ & $0.08^{\mathrm{E}} \pm 0.02$ \\
\hline & $\begin{array}{l}\text { Temperature } \\
\left({ }^{\circ} \mathrm{C}\right)\end{array}$ & $\operatorname{Rg} 2(S)$ & $\operatorname{Rg} 2(\mathrm{R})$ & $\operatorname{Rg} 3(S)$ & $\operatorname{Rg} 3(\mathrm{R})$ & Rh1(S) & $\operatorname{Rh} 2(S)$ & Sum & \\
\hline & Control & $0.17^{\mathrm{CD}} \pm 0.01$ & $0.09^{\mathrm{B}} \pm 0.01$ & $\mathrm{ND}^{\mathrm{E}}$ & $\mathrm{ND}^{\mathrm{C}}$ & $0.10^{\mathrm{C}} \pm 0.02$ & ND & $15.77^{\mathrm{bA}} \pm 0.02$ & \\
\hline & 160 & $0.16^{\mathrm{D}} \pm 0.07$ & $0.09^{\mathrm{B}} \pm 0.07$ & $\mathrm{ND}^{\mathrm{E}}$ & $\mathrm{ND}^{\mathrm{C}}$ & $0.05^{C} \pm 0.03$ & ND & $13.89^{b C} \pm 0.04$ & \\
\hline & 170 & $0.24^{C} \pm 0.06$ & $0.19^{\mathrm{A}} \pm 0.06$ & $0.08^{\mathrm{D}} \pm 0.02$ & $0.08^{\mathrm{AB}} \pm 0.03$ & $0.10^{C} \pm 0.00$ & ND & $14.72^{\mathrm{bcB}} \pm 0.04$ & \\
\hline & 180 & $0.26^{\mathrm{BC}} \pm 0.04$ & $0.10^{\mathrm{B}} \pm 0.04$ & $0.19 C_{ \pm 0.01}$ & $0.07^{\mathrm{B}} \pm 0.03$ & $0.22^{\mathrm{B}} \pm 0.00$ & ND & $11.06^{\mathrm{cD}} \pm 0.03$ & \\
\hline & 190 & $0.39 \mathrm{~A} \pm 0.06$ & $0.10^{\mathrm{B}} \pm 0.03$ & $0.45^{\mathrm{A}} \pm 0.01$ & $0.11^{\mathrm{AB}} \pm 0.04$ & $0.34^{\mathrm{A}} \pm 0.06$ & ND & $6.18^{\mathrm{aE}} \pm 0.03$ & \\
\hline & 200 & $0.27^{\mathrm{BC}} \pm 0.04$ & $0.00^{C} \pm 0.00$ & $0.36^{\mathrm{B}} \pm 0.02$ & $0.13^{\mathrm{A}} \pm 0.03$ & $0.24^{\mathrm{B}} \pm 0.04$ & ND & $2.40^{\mathrm{aF}} \pm 0.05$ & \\
\hline $\begin{array}{l}\text { Size } \\
(\mu \mathrm{m})\end{array}$ & $\begin{array}{l}\text { Temperature } \\
\left({ }^{\circ} \mathrm{C}\right)\end{array}$ & $\operatorname{Rg} 1$ & $\operatorname{Re}$ & Rf & $\mathrm{Rb} 1$ & Rc & $\mathrm{Rb} 2$ & $\mathrm{Rb} 3$ & $\mathrm{Rd}$ \\
\hline \multirow{9}{*}{$20-30$} & Control & $3.28^{\mathrm{C}} \pm 0.04$ & $2.68^{\mathrm{B}} \pm 0.02$ & $0.81^{\mathrm{AB}} \pm 0.70$ & $3.60^{\mathrm{B}} \pm 0.06$ & $0.90^{\mathrm{D}} \pm 0.02$ & $0.78^{\mathrm{D}} \pm 0.02$ & $0.19^{\mathrm{AB}_{ \pm}} 0.01$ & $0.28^{\mathrm{A}} \pm 0.02$ \\
\hline & 160 & $3.87^{\mathrm{A}} \pm 0.22$ & $2.90^{\mathrm{A}} \pm 0.12$ & $0.92^{\mathrm{A}} \pm 0.06$ & $4.64^{\mathrm{A}} \pm 0.32$ & $1.30^{\mathrm{B}} \pm 0.03$ & $0.99^{\mathrm{B}} \pm 0.06$ & $0.20^{\mathrm{AB}} \pm 0.03$ & $0.31^{\mathrm{A}} \pm 0.05$ \\
\hline & 170 & $3.62^{\mathrm{B}} \pm 0.08$ & $2.55^{\mathrm{C}} \pm 0.05$ & $0.94^{\mathrm{A}} \pm 0.02$ & $4.51^{\mathrm{A}_{ \pm}} 0.04$ & $1.42^{\mathrm{A}} \pm 0.08$ & $1.12^{\mathrm{A}} \pm 0.03$ & $0.24^{\mathrm{A}} \pm 0.06$ & $0.29^{\mathrm{A}} \pm 0.03$ \\
\hline & 180 & $2.17^{\mathrm{D}} \pm 0.07$ & $2.08^{\mathrm{D}} \pm 0.08$ & $0.73^{\mathrm{AB}} \pm 0.03$ & $3.08^{C} \pm 0.16$ & $1.05^{\mathrm{C}} \pm 0.00$ & $0.84^{\mathrm{C}} \pm 0.02$ & $0.14^{\mathrm{B}} \pm 0.01$ & $0.23^{\mathrm{B}} \pm 0.02$ \\
\hline & 190 & $0.61^{\mathrm{E}} \pm 0.00$ & $0.70^{\mathrm{E}} \pm 0.00$ & $0.51^{\mathrm{AB}_{ \pm}} 0.00$ & $1.04^{\mathrm{D}} \pm 0.08$ & $0.42^{\mathrm{E}} \pm 0.02$ & $0.36^{\mathrm{E}} \pm 0.02$ & $0.04^{\mathrm{C}} \pm 0.01$ & $0.10^{C} \pm 0.00$ \\
\hline & 200 & $0.08^{\mathrm{F}} \pm 0.00$ & $0.20^{\mathrm{F}} \pm 0.00$ & $0.23^{\mathrm{B}} \pm 0.01$ & $0.29 \mathrm{E}_{ \pm 0} 0.03$ & $0.14^{\mathrm{F}} \pm 0.02$ & $0.14^{\mathrm{F}} \pm 0.03$ & $0.03^{C} \pm 0.04$ & $0.06^{\mathrm{C}} \pm 0.02$ \\
\hline & $\begin{array}{l}\text { Temperature } \\
\left({ }^{\circ} \mathrm{C}\right)\end{array}$ & $\operatorname{Rg} 2(S)$ & $\operatorname{Rg} 2(\mathrm{R})$ & $\operatorname{Rg} 3(S)$ & $\operatorname{Rg} 3(\mathrm{R})$ & $\operatorname{Rh1}(S)$ & Rh2(S) & Sum & \\
\hline & Control & $0.06^{\mathrm{C}} \pm 0.02$ & $0.06^{\mathrm{C}} \pm 0.01$ & $\mathrm{ND}^{\mathrm{D}}$ & $\mathrm{ND}^{\mathrm{C}}$ & $0.10^{\mathrm{C}} \pm 0.01$ & ND & $12.75^{\mathrm{c}} \pm 0.07$ & \\
\hline & 160 & $0.23^{\mathrm{B}} \pm 0.05$ & $0.22^{\mathrm{A}} \pm 0.09$ & $0.04^{\mathrm{D}} \pm 0.01$ & $0.11^{\mathrm{A}} \pm 0.01$ & $0.05^{\mathrm{D}} \pm 0.03$ & ND & $15.77^{\mathrm{aA}} \pm 0.08$ & \\
\hline
\end{tabular}




\begin{tabular}{|c|c|c|c|c|c|c|c|c|c|}
\hline & 170 & $0.24^{\mathrm{B}} \pm 0.06$ & $0.23^{\mathrm{A}} \pm 0.06$ & $0.12^{\complement} \pm 0.03$ & $0.10^{\mathrm{A}} \pm 0.03$ & $0.14^{\complement} \pm 0.05$ & ND & $15.52^{\mathrm{aB}} \pm 0.04$ & \\
\hline & 180 & $0.32^{\mathrm{A}} \pm 0.02$ & $0.16^{\mathrm{AB}_{ \pm}} \pm .01$ & $0.19^{\mathrm{B}} \pm 0.02$ & $0.05^{\mathrm{B}} \pm 0.01$ & $0.22^{\mathrm{B}} \pm 0.03$ & ND & $11.26^{\mathrm{bcD}} \pm 0.03$ & \\
\hline & 190 & $0.33^{\mathrm{A}} \pm 0.02$ & $0.08^{\mathrm{BC}} \pm 0.00$ & $0.36^{\mathrm{A}} \pm 0.01$ & $0.11^{\mathrm{A}} \pm 0.04$ & $0.33^{\mathrm{A}} \pm 0.01$ & ND & $4.97 \mathrm{bE} \pm 0.01$ & \\
\hline & 200 & $0.28^{\mathrm{AB}_{ \pm}} \pm 0.02$ & $\mathrm{ND}^{\mathrm{C}}$ & $0.40^{\mathrm{A}} \pm 0.05$ & $0.13^{\mathrm{A}} \pm 0.02$ & $0.23^{\mathrm{B}} \pm 0.02$ & ND & $2.22^{\mathrm{aF}} \pm 0.02$ & \\
\hline $\begin{array}{l}\text { Size } \\
(\mu \mathrm{m})\end{array}$ & $\begin{array}{l}\text { Temperature } \\
\left({ }^{\circ} \mathrm{C}\right)\end{array}$ & Rg1 & $\operatorname{Re}$ & Rf & $\mathrm{Rb} 1$ & Rc & $\mathrm{Rb} 2$ & $\mathrm{Rb} 3$ & $\mathrm{Rd}$ \\
\hline \multirow{13}{*}{$30-40$} & Control & $3.93^{\mathrm{A}} \pm 0.08$ & $3.24^{\mathrm{A}} \pm 0.04$ & $1.13^{\mathrm{A}} \pm 0.31$ & $4.60^{\mathrm{A}} \pm 0.28$ & $1.57^{\mathrm{A}} \pm 0.16$ & $1.33^{\mathrm{A}} \pm 0.17$ & $0.19^{\mathrm{A}} \pm 0.04$ & $0.42^{\mathrm{A}} \pm 0.07$ \\
\hline & 160 & $3.31^{\mathrm{B}} \pm 0.03$ & $2.35^{\mathrm{C}} \pm 0.07$ & $0.75^{\mathrm{B}} \pm 0.04$ & $3.39{ }^{C} \pm 0.05$ & $1.26^{\mathrm{B}} \pm 0.03$ & $1.02^{\mathrm{B}} \pm 0.04$ & $0.19^{\mathrm{A}} \pm 0.01$ & $0.16^{\mathrm{CD}_{ \pm}} \pm .03$ \\
\hline & 170 & $3.10^{C} \pm 0.00$ & $2.73^{\mathrm{B}} \pm 0.10$ & $0.76^{\mathrm{B}} \pm 0.00$ & $4.18^{\mathrm{B}} \pm 0.08$ & $1.34^{\mathrm{B}} \pm 0.00$ & $1.06^{\mathrm{B}} \pm 0.04$ & $0.13^{\mathrm{AB}_{ \pm} 0.01}$ & $0.21^{\mathrm{BC}} \pm 0.06$ \\
\hline & 180 & $2.14^{\mathrm{D}} \pm 0.05$ & $2.26^{C} \pm 0.07$ & $0.68^{\mathrm{B}} \pm 0.00$ & $3.39 C_{ \pm 0.07}$ & $1.26^{\mathrm{B}} \pm 0.09$ & $1.06^{\mathrm{B}} \pm 0.06$ & $0.15^{\mathrm{AB}} \pm 0.04$ & $0.26^{\mathrm{B}} \pm 0.03$ \\
\hline & 190 & $0.53^{\mathrm{E}} \pm 0.02$ & $0.72^{\mathrm{D}} \pm 0.03$ & $0.46^{\mathrm{BC}} \pm 0.05$ & $0.99 \mathrm{D} \pm 0.03$ & $0.44^{C} \pm 0.06$ & $0.38^{C} \pm 0.03$ & $0.08^{\mathrm{B}} \pm 0.08$ & $0.11^{\mathrm{D}} \pm 0.00$ \\
\hline & 200 & $0.06^{\mathrm{F}} \pm 0.00$ & $0.12^{\mathrm{E}} \pm 0.00$ & $0.24^{\mathrm{C}} \pm 0.00$ & $0.21^{\mathrm{E}} \pm 0.00$ & $0.09 \mathrm{D}^{\mathrm{D}} \pm 0.05$ & $0.11^{\mathrm{D}} \pm 0.01$ & $0.05^{\mathrm{B}} \pm 0.03$ & $0.07^{\mathrm{D}} \pm 0.00$ \\
\hline & $\begin{array}{l}\text { Temperature } \\
\left({ }^{\circ} \mathrm{C}\right)\end{array}$ & $\operatorname{Rg} 2(S)$ & $\operatorname{Rg} 2(\mathrm{R})$ & $\operatorname{Rg} 3(S)$ & $\operatorname{Rg} 3(\mathrm{R})$ & Rh1(S) & Rh2(S) & Sum & \\
\hline & Control & $0.20^{\mathrm{AB}} \pm 0.08$ & $0.11^{\mathrm{BC}} \pm 0.12$ & $\mathrm{ND}^{\mathrm{E}}$ & $\mathrm{ND}^{\mathrm{C}}$ & $0.05^{\mathrm{B}} \pm 0.09$ & ND & $16.77^{\mathrm{aA}} \pm 0.10$ & \\
\hline & 160 & $0.16^{\mathrm{B}} \pm 0.01$ & $0.18^{\mathrm{AB}_{ \pm}} 0.00$ & $0.01^{\mathrm{E}_{ \pm}} 0.00$ & $0.05^{\mathrm{B}} \pm 0.00$ & $0.03^{\mathrm{B}} \pm 0.00$ & ND & $12.84^{\mathrm{c} C} \pm 0.02$ & \\
\hline & 170 & $0.27^{\mathrm{AB}} \pm 0.07$ & $0.28^{\mathrm{A}} \pm 0.08$ & $0.08^{\mathrm{D}} \pm 0.03$ & $0.07^{\mathrm{AB}} \pm 0.03$ & $0.08^{\mathrm{B}} \pm 0.03$ & ND & $14.30^{\mathrm{cB}} \pm 0.04$ & \\
\hline & 180 & $0.32^{\mathrm{A}} \pm 0.01$ & $0.09^{\mathrm{BC}} \pm 0.00$ & $0.18^{\mathrm{C}} \pm 0.00$ & $0.05^{\mathrm{B}} \pm 0.00$ & $0.20^{\mathrm{A}} \pm 0.04$ & $\mathrm{ND}$ & $12.05^{\mathrm{a} D} \pm 0.03$ & \\
\hline & 190 & $0.30^{\mathrm{A}} \pm 0.06$ & $0.08^{\mathrm{BC}} \pm 0.03$ & $0.35^{\mathrm{B}} \pm 0.01$ & $0.08^{\mathrm{A}} \pm 0.00$ & $0.27^{\mathrm{A}} \pm 0.00$ & $\mathrm{ND}$ & $4.78^{\mathrm{bE}} \pm 0.03$ & \\
\hline & 200 & $0.22^{\mathrm{AB}_{ \pm}} 0.01$ & $0.00^{C} \pm 0.00$ & $0.41^{\mathrm{A}} \pm 0.02$ & $0.10^{\mathrm{A}} \pm 0.00$ & $0.27^{\mathrm{A}} \pm 0.01$ & ND & $1.95^{\mathrm{abF}} \pm 0.01$ & \\
\hline $\begin{array}{l}\text { Size } \\
(\mu \mathrm{m})\end{array}$ & $\begin{array}{l}\text { Temperature } \\
\left({ }^{\circ} \mathrm{C}\right)\end{array}$ & $\operatorname{Rg1}$ & $\operatorname{Re}$ & $\mathrm{Rf}$ & $\mathrm{Rb} 1$ & Rc & $\mathrm{Rb} 2$ & $\mathrm{Rb} 3$ & $\mathrm{Rd}$ \\
\hline \multirow{13}{*}{$40-50$} & Control & $3.14^{\mathrm{B}} \pm 0.02$ & $2.70^{\mathrm{B}} \pm 0.01$ & $1.05^{\mathrm{A}} \pm 0.03$ & $3.23^{\mathrm{C}} \pm 0.14$ & $0.73^{\mathrm{D}} \pm 0.06$ & $0.66^{\mathrm{D}} \pm 0.04$ & $0.17^{\mathrm{A}} \pm 0.01$ & $0.25^{\mathrm{AB}_{ \pm}} 0.02$ \\
\hline & 160 & $3.56^{\mathrm{A}} \pm 0.12$ & $2.86^{\mathrm{A}} \pm 0.02$ & $0.85^{\mathrm{B}} \pm 0.02$ & $4.33^{\mathrm{A}} \pm 0.02$ & $1.34^{\mathrm{A}} \pm 0.03$ & $1.11^{\mathrm{A}} \pm 0.02$ & $0.24^{\mathrm{A}} \pm 0.04$ & $0.27^{\mathrm{A}} \pm 0.01$ \\
\hline & 170 & $2.98^{C} \pm 0.00$ & $2.37 C_{ \pm 0.06}$ & $0.85^{\mathrm{B}} \pm 0.01$ & $3.75^{\mathrm{B}} \pm 0.07$ & $1.23^{\mathrm{B}} \pm 0.00$ & $0.98^{\mathrm{B}} \pm 0.00$ & $0.20^{\mathrm{A}} \pm 0.08$ & $0.22^{\mathrm{BC}} \pm 0.03$ \\
\hline & 180 & $2.16^{\mathrm{D} \pm 0.03}$ & $1.85^{\mathrm{D}} \pm 0.01$ & $0.76^{\mathrm{C}} \pm 0.01$ & $2.95^{\mathrm{D}} \pm 0.05$ & $1.044_{ \pm 0.06}$ & $0.91^{C_{ \pm}} \pm .03$ & $0.18^{\mathrm{A}} \pm 0.07$ & $0.19 C_{ \pm 0.01}$ \\
\hline & 190 & $0.77^{\mathrm{E}} \pm 0.02$ & $0.82^{\mathrm{E}} \pm 0.03$ & $0.54^{\mathrm{D}} \pm 0.00$ & $1.28^{\mathrm{E}} \pm 0.00$ & $0.46^{\mathrm{E}} \pm 0.01$ & $0.44^{\mathrm{E}} \pm 0.03$ & $0.15^{\mathrm{A}} \pm 0.01$ & $0.13^{\mathrm{D}} \pm 0.02$ \\
\hline & 200 & $0.10^{\mathrm{F}} \pm 0.02$ & $0.17^{\mathrm{F}_{ \pm}} 0.01$ & $0.19^{\mathrm{E}} \pm 0.00$ & $0.28^{\mathrm{F}} \pm 0.01$ & $0.10^{\mathrm{F}} \pm 0.00$ & $0.15^{\mathrm{F}} \pm 0.00$ & $0.03^{\mathrm{B}} \pm 0.01$ & $0.08^{\mathrm{E}} \pm 0.01$ \\
\hline & $\begin{array}{l}\text { Temperature } \\
\left({ }^{\circ} \mathrm{C}\right)\end{array}$ & $\operatorname{Rg} 2(S)$ & $\operatorname{Rg} 2(\mathrm{R})$ & $\operatorname{Rg} 3(S)$ & $\operatorname{Rg} 3(\mathrm{R})$ & Rh1(S) & Rh2(S) & Sum & \\
\hline & Control & $0.07^{\mathrm{B}} \pm 0.01$ & $0.02^{\mathrm{B}} \pm 0.01$ & $\mathrm{ND}^{\mathrm{E}}$ & $\mathrm{ND}^{\mathrm{C}}$ & $0.11^{\mathrm{B}} \pm 0.01$ & ND & $12.13^{\mathrm{c}} \pm 0.03$ & \\
\hline & 160 & $0.20^{\mathrm{A}} \pm 0.01$ & $0.16^{\mathrm{A}} \pm 0.06$ & $0.03^{\mathrm{D}} \pm 0.00$ & $0.09^{\mathrm{B}} \pm 0.00$ & $0.03^{C} \pm 0.01$ & ND & $15.06^{\mathrm{aA}} \pm 0.02$ & \\
\hline & 170 & $0.24^{\mathrm{A}} \pm 0.05$ & $0.20^{\mathrm{A}} \pm 0.01$ & $0.09 C^{C} \pm 0.02$ & $0.07^{\mathrm{B}} \pm 0.01$ & $0.11^{\mathrm{B}} \pm 0.03$ & ND & $13.29 \mathrm{~dB}_{ \pm 0} .02$ & \\
\hline & 180 & $0.27^{\mathrm{A}} \pm 0.08$ & $0.16^{\mathrm{A}} \pm 0.04$ & $0.19^{\mathrm{B}} \pm 0.02$ & $0.05^{\mathrm{B}} \pm 0.05$ & $0.23^{\mathrm{A}} \pm 0.06$ & ND & $10.93^{\mathrm{cD}} \pm 0.04$ & \\
\hline & 190 & $0.28^{\mathrm{A}} \pm 0.01$ & $0.06^{\mathrm{A}} \pm 0.01$ & $0.39^{\mathrm{A}} \pm 0.01$ & $0.09^{\mathrm{B}} \pm 0.01$ & $0.299^{\mathrm{A}} \pm 0.01$ & ND & $5.67^{\mathrm{a}} \pm 0.01$ & \\
\hline & 200 & $0.23^{\mathrm{A}} \pm 0.02$ & $0.08^{\mathrm{A}} \pm 0.00$ & $0.38^{\mathrm{A}} \pm 0.01$ & $0.12^{\mathrm{A}} \pm 0.03$ & $0.17^{\mathrm{B}} \pm 0.01$ & ND & $2.07^{a F} \pm 0.01$ & \\
\hline $\begin{array}{l}\text { Size } \\
(\mu \mathrm{m})\end{array}$ & $\begin{array}{l}\text { Temperature } \\
\left({ }^{\circ} \mathrm{C}\right)\end{array}$ & Rg1 & $\operatorname{Re}$ & Rf & $\mathrm{Rb} 1$ & Rc & $\mathrm{Rb} 2$ & $\mathrm{Rb} 3$ & $\mathrm{Rd}$ \\
\hline \multirow{13}{*}{$>50$} & Control & $3.81^{\mathrm{A}} \pm 0.10$ & $3.27^{\mathrm{A}} \pm 0.05$ & $1.50^{\mathrm{A}} \pm 0.05$ & $4.20^{\mathrm{B}} \pm 0.11$ & $1.10^{\mathrm{B}} \pm 0.05$ & $0.94^{\mathrm{B}} \pm 0.05$ & $0.20^{\mathrm{AB}} \pm 0.03$ & $0.29^{\mathrm{A}} \pm 0.03$ \\
\hline & 160 & $3.89^{\mathrm{A}} \pm 0.00$ & $2.26^{C} \pm 0.04$ & $0.87^{\mathrm{BC}} \pm 0.02$ & $3.97 C_{ \pm 0.07}$ & $1.16^{\mathrm{B}} \pm 0.03$ & $0.93^{\mathrm{B}} \pm 0.03$ & $0.19^{\mathrm{B}} \pm 0.02$ & $0.24^{\mathrm{B}} \pm 0.02$ \\
\hline & 170 & $3.25^{\mathrm{B}} \pm 0.03$ & $2.86^{\mathrm{B}} \pm 0.03$ & $0.89^{\mathrm{B}} \pm 0.06$ & $4.37^{\mathrm{A}} \pm 0.00$ & $1.40^{\mathrm{A}} \pm 0.01$ & $1.14^{\mathrm{A}} \pm 0.00$ & $0.29^{\mathrm{A}} \pm 0.01$ & $0.28^{\mathrm{AB}} \pm 0.00$ \\
\hline & 180 & $2.22^{\mathrm{C}} \pm 0.01$ & $1.98^{\mathrm{D}} \pm 0.05$ & $0.81^{C} \pm 0.00$ & $3.14^{\mathrm{D}} \pm 0.03$ & $1.14^{\mathrm{B}} \pm 0.07$ & $0.96^{\mathrm{B}} \pm 0.03$ & $0.20^{\mathrm{AB}} \pm 0.06$ & $0.27^{\mathrm{AB}} \pm 0.03$ \\
\hline & 190 & $0.63^{\mathrm{D}} \pm 0.01$ & $0.66^{\mathrm{E}} \pm 0.02$ & $0.50^{\mathrm{D}} \pm 0.03$ & $1.04^{\mathrm{E}} \pm 0.05$ & $0.39 C_{ \pm 0.00}$ & $0.377_{ \pm 0.02}$ & $0.13^{\mathrm{BC}} \pm 0.07$ & $0.11^{C_{ \pm}} \pm 0.00$ \\
\hline & 200 & $0.06^{\mathrm{E}} \pm 0.00$ & $0.11^{\mathrm{F}} \pm 0.00$ & $0.19^{\mathrm{E}} \pm 0.02$ & $0.17^{\mathrm{F}} \pm 0.02$ & $0.06^{\mathrm{D}} \pm 0.00$ & $0.11^{\mathrm{D}} \pm 0.00$ & $0.05^{\mathrm{C}} \pm 0.00$ & $0.077_{ \pm 0.01}$ \\
\hline & $\begin{array}{l}\text { Temperature } \\
\left({ }^{\circ} \mathrm{C}\right)\end{array}$ & $\operatorname{Rg} 2(S)$ & $\operatorname{Rg} 2(\mathrm{R})$ & $\operatorname{Rg} 3(S)$ & $\operatorname{Rg} 3(\mathrm{R})$ & Rh1(S) & Rh2(S) & Sum & \\
\hline & Control & $0.16^{\mathrm{B}} \pm 0.03$ & $0.09^{\mathrm{B}} \pm 0.01$ & $\mathrm{ND}^{\mathrm{D}}$ & $\mathrm{ND}^{\mathrm{B}}$ & $0.11^{C_{ \pm}} 0.02$ & ND & $15.66^{\mathrm{bA}} \pm 0.04$ & \\
\hline & 160 & $0.17^{\mathrm{B}} \pm 0.04$ & $0.21^{\mathrm{A}} \pm 0.01$ & $0.03^{\mathrm{D}} \pm 0.01$ & $0.10^{\mathrm{A}} \pm 0.01$ & $0.08^{C} \pm 0.03$ & ND & $14.09^{b C} \pm 0.02$ & \\
\hline & 170 & $0.23^{\mathrm{B}} \pm 0.01$ & $0.20^{\mathrm{A}} \pm 0.08$ & $0.11^{C_{ \pm}} \pm 0.01$ & $0.12^{\mathrm{A}} \pm 0.00$ & $0.099_{ \pm 0.01}$ & ND & $15.22^{\mathrm{abB}} \pm 0.02$ & \\
\hline & 180 & $0.33^{\mathrm{A}} \pm 0.04$ & $0.17^{\mathrm{A}} \pm 0.02$ & $0.26^{\mathrm{B}} \pm 0.02$ & $0.12^{\mathrm{A}} \pm 0.00$ & $0.21^{\mathrm{B}} \pm 0.00$ & ND & $11.82^{\mathrm{abD}_{ \pm} 0.02}$ & \\
\hline & 190 & $0.30^{\mathrm{A}} \pm 0.06$ & $0.10^{\mathrm{B}} \pm 0.02$ & $0.36^{\mathrm{A}} \pm 0.02$ & $0.10^{\mathrm{A}} \pm 0.02$ & $0.32^{\mathrm{A}} \pm 0.04$ & ND & $5.03^{\mathrm{bE}} \pm 0.02$ & \\
\hline & 200 & $0.19^{\mathrm{B}} \pm 0.04$ & $\mathrm{NDC}^{\mathrm{C}}$ & $0.34^{\mathrm{A}} \pm 0.03$ & $0.10^{\mathrm{A}} \pm 0.00$ & $0.18^{\mathrm{B}} \pm 0.04$ & ND & $1.64^{\mathrm{bF} \pm 0.01}$ & \\
\hline
\end{tabular}




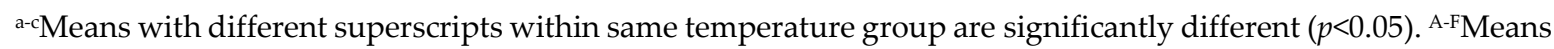
with different superscripts within same size group are significantly different $(p<0.05) .{ }^{1)}$ After cryogenic milling of ginseng samples in different conditions, samples were classified based on their particle sizes. One treatment condition was selected for each size range, and particle-size based selected samples were roasted and pulverized by cryogenic milling.

\section{Conclusion}

In this study, ginseng was pretreated at different roasting temperatures and was ground using different cryogenic milling conditions to reduce particles size and ultimately, increase water solubility and bioactive components. The particle size of ginseng was found to be significantly smaller in all roasting pretreatments compared to that in the control (without roasting pretreatment) $(p<0.05)$. A decreasing trend of particle size was revealed with increasing roasting temperature. When ginseng powder of $10-20 \mu \mathrm{m}$ size was roasted at $170{ }^{\circ} \mathrm{C}$, smallest particle size was found to be 5.41 $\mu \mathrm{m}$. However, we found that the particle size of the ginseng increased due to flocculation when roasted above $180^{\circ} \mathrm{C}$. Similar pattern was observed with other particle sizes $(>20 \mu \mathrm{m})$ above $190{ }^{\circ} \mathrm{C}$. On comparing the physicochemical properties based on particle size of ginseng samples; WSI, antioxidant activity, TPC, and total polysaccharide contents were found to increase with decrease in particle size. On analyzing WSI, TPC, and total polysaccharide content based on roasting temperature, an increase in their levels was observed with rise in roasting temperature below $180^{\circ} \mathrm{C}$. However, three contents were observed to decrease at $190^{\circ} \mathrm{C}$ and above. Such trend might be due to loss of internal components in ginseng caused at high temperature. In this study, the powder appeared black in color when roasted at $190{ }^{\circ} \mathrm{C}$ and above, which may be caused by burning. It is believed that high roasting temperature causes loss of bioactive substances in ginseng. Antioxidant activities showed a trend of increasing values with decrease in particle size $(p>0.05)$. Furthermore, all roasted samples showed significantly increased antioxidant activities compared to that of the controls $(p<0.05)$. Additionally, we found that a higher roasting temperature increases the antioxidant activities of ginseng. Total ginsenoside contents showed no alterations based on particle size and roasting temperature. However, total ginsenosides showed a sharp decrease when samples were roasted at $190{ }^{\circ} \mathrm{C}$ and above. Non-polar ginsenosides such as $\operatorname{Rg} 2(\mathrm{~S}), \operatorname{Rg} 2(\mathrm{R}), \operatorname{Rg} 3(\mathrm{~S}), \operatorname{Rg} 3(\mathrm{R})$, and $\mathrm{Rh} 1(\mathrm{~S})$ tended to increase with increase in roasting temperature. Overall, we reveal that roasting within certain temperature range followed by cryogenic milling is an effective method to reduce particle size, and increase water solubility and bioactive components of ginseng.

Author Contributions: H. Jeong performed the experiments and wrote original draft; D. H. Park performed the experiments; H. G. Seo and M.-J. Choi provided technical and material support; Y. Cho interpreted the data and critically revised the manuscript.

Funding: This work was supported by Korea Institute of Planning and Evaluation for Technology in Food, Agriculture and Forestry (iPET) through High Value-added Food Technology Development Program, funded by Ministry of Agriculture, Food and Rural Affairs (MAFRA) (318028-03). This work was also supported by the KU Research Professor Program of Konkuk University.

Conflicts of Interest: The authors declare no conflict of interest.

\section{References}

1. Yun, T.K. Brief introduction of Panax ginseng C.A. Meyer. J Korean Med Sci. 2001, 16, S3-S5; DOI: 10.3346/jkms.2001.16.S.S3.

2. Park, S.Y.; Lee, K.Y.; Cho, Y.J.; Park, B.K.; Kim, K.J.; Lee, N.R.; Kim, D.G.; Kim, Y.H.; Hahn, T.W. Efficacy of orally administered ginseng stem and leaf in chickens. Korean J. Vet. Res. 2015, 55(1), 1-7; DOI: 10.14405/kjvr.2015.55.1.1.

3. Li, X.G. Studies on the transforming mechanism of amino acid components in ginseng in the course of ginseng process. Korean J. Ginseng Sci. 1992, 16(1), 64-67.

4. Kang, K.S.; Yokozawa, T.; Yamabe, N.; Kim, H. Y.; Park, J.H. ESR study on the structure and hydroxyl radicalscavenging activity relationships of ginsenosides isolate from Panax ginseng C. A. Meyer. Biol. Pharm. Bull. 2007, 30(5), 917-921; DOI: 10.1248/bpb.30.917. 
5. Park, C.K.; Jeon, B.S.; Yang, J.W. The chemical components of Korean ginseng. Food Ind Nutr. 2003, 8(2), 1023.

6. Lee, S.B.; Yoo, S.H.; Ganesan, P.; Kwak, H.S. Physicochemical and antioxidative properties of Korean nano powdered white ginseng. Int J Food Sci Tech. 2013, 48(10), 2159-2165; DOI: 10.1111/IJFS.12200.

7. Choi, K.T. Botanical characteristics, pharmacological effects and medicinal components of Korean Panax ginseng C A Meyer. Acta Pharmacologica Sinica 2008, 29(9), 1109-1118; DOI: 10.1111/J.1745-7254.2008.00869.x.

8. Lu, J.M.; Yao, Q.; Chen, C. Ginseng compounds: an update on their molecular mechanisms and medical applications. Curr. Vasc. Pharmacol. 2009, 7(3), 293-302; DOI: 10.2174/157016109788340767.

9. Ren, G.; Chen, F. Drying of American ginseng (Panax quinquefolius) roots by microwave-hot air combination. J. Food Eng. 1998, 35(4), 433-443; DOI: 10.1016/S0260-8774(98)00030-2.

10. Davidson, V.J.; Martynenko, A.I.; Parhar, N.K.; Sidahmed, M. Forced-air drying of ginseng root: pilot-scale control system for three-stage process. Dry Technol 2009, 27(3), 451-458; DOI: 10.1080/07373930802683757.

11. Sacilik, K. Effect of drying methods on thin-layer drying characteristics of hull-less seed pumplin (Cucurbita pepo L.). J. Food Eng. 2007, 79(1), 23-30; DOI: 10.1016/j.jfoodeng.2006.01.023.

12. Choi, K.O.; Lee, I.; Park, S.Y.R.; Kim, D.E.; Lim, J.D.; Kang, W.S.; Ko, S.H. Ultrafine Angelica gigas powder normalizes ovarian hormone levels and has antiosteoporosis properties in ovariectomized rats: particle size effect. J. Med. Food 2012, 15(10), 863-872; DOI: 10.1089/jmf.2011.2047.

13. Suh, C.S.; Chun, J.K. Relationships among the roasting conditions, colors and extractable solid content of roasted barley. Korean J. Food Sci. Technol. 1981, 13(4), 334-339.

14. Jeon, E.J.; Kim, K.Y.; Lee, J.E.; Catherine, W.; Kwon, J.H. Monitoring of Roasting Conditions for the Functional Properties of Lateral Root of Red Ginseng. Korean J. Food Preserv. 2008, 15(3), 396-404.

15. Park, M.H.; Kim, K.C. Changes in Physicochemical Components of Ginseng Marc by Roasting Process. Korean J. Ginseng Sci. 1995, 19(2), 144-152.

16. Sivetz, M.; Desrosier, N.W. Coffee technology. United States Department of Agriculture National Agricultural Library. Westport, CT: Avi, USA, 1979; pp 716.

17. Yoon, S.K.; Kim, W.J. Effects of roasting conditions on quality and yields of barley tea. Korean J. Food Sci. Technol. 1989, 21(4), 575-582.

18. Lee, B.G.; Lee, K.Y.; Jorge, S.; Jorge, R.; Baek, H.; Min, J.H.; Kang, W.S. Ultrafine powderization using low temperature turbo mill to improve water solubility of red ginseng powder. In 2012 12th IEEE International Conference on Nanotechnology (IEEE-NANO) 2012, 1-4; DOI: 10.1109/NANO.2012.6321975.

19. Park, S.K.; Kim, Y.K.; Youn, H.S.; Lee, M.Y. Application of Nanotechnology to Korean Black-Red Ginseng: Solubility Enhancement by Particle Size Reduction. Mol Cell Toxicol 2008, 4(1), 52-60.

20. Manohar, B.; Sridhar, B.S. Size and Shape characterization of conventionally and cryogenically ground turmeric (Curcuma domestica) particles. Powder Technol. 2001, 120, 292-297; DOI: 10.116/S0032-5910(01)002844.

21. Kim, H.Y.; Seo, H.I.; Ko, J.Y.; Kim, J.I.; Lee, J.S.; Song, S.B.; Jung, T.W.; Kim, K.Y.; Kwak, D.Y.; Oh, I.S.; Jeong, H.S.; Woo, K.S. Physicochemical Characteristics of the Sorhum(Sorghum bicolor L. Moench) Powder following Low Temperature-Microparticulation. Korean J. Food E Nutr. 2012, 25(3), 656-663.

22. Blois, M.S. Antioxidant determinations by the use of a stable free radical. Nature 1958, 181, 1199-1200; DOI: 10.1038/1811199a0.

23. Ough, C.S.; American, M.A. Methods for analysis of musts and wine; Wiley \& Sons: New York, NY, USA, 1988; pp 176-180.

24. Dubois, M.; Gillers, K.A.; Hamilton, J.K.; Rebers, P.A.; Smith, J. Colorimetric method for determination of sugar and related substance. Anal. Chem. 1956, 28(3), 350-356; DOI: 10.1021/ac60111A017.

25. Do, J.H.; Lee, H.O.; Lee, S.K.; Jang, J.K.; Lee, S.D.; Sung, H.S. Colorimetric Determination of Acidic Polysaccharide from Panax ginseng, its Extraction Condition and Stability). J. Ginseng Res. 1993, 17(2), 139144.

26. Kim W.; Choi S.; Kerr W.; Johnson J.; Gaines, C. Effect of heating temperature on particle size distribution in hard and soft wheat flour. J. Cereal Sci. 2004, 40(1), 9-16. DOI: 10.1016/J.JCS.2004.04.005.

27. Tkacova, K.; N, Stevulova. Selected problems of the dispersity analysis of milled ultrafine powder. Freiberger Forsclungshefte AA841 (Partikeltechnologie), 1998, 841, 14-25.

28. Lee, J.S.; Lee, H.S. Effect of grinding methods on particle size and crystalline structure of copper phthalocyanine. J Ind Eng Chem. 2007, 18(1), 41-47. 
29. Uhm, Y.R.; Kim, J.W.; Jung, J.W.; Rhee, C.K. The fabrication of PVA polymer coated on the surface of B4C nanocomposite by high energy ball mill. J. Korean Powder Metall. 2009, 16(2), 110-114; DOI: 10.4150/KPMI.2009.16.2.110.

30. Seong, B.J.; Kim, S.I.; Jee, M.G.; Kim, S.D.; Kwon, A.R.; Kim, H.H.; Hwang, Y.G.; Lee, K.S. Physicochemical characteristics according to the roasting conditions and grinding grade for the development of drip type red ginseng. J Korean Soc Food Sci Nutr 2018, 47(3), 309-319.

31. Park, M.H.; Kim, Y.H.; Yang, J.W.; Kim, K.C. Separation and characteristics of the extract solution from ginseng marc by roasting process. Korean Journal of Ginseng Science (Korea Republic) 1995, 19(2), 153-159.

32. Jo, G.S., Sin, J.S.; Kim, J.H. Measurement of particle size and particle size distribution. Polymer Sci Technol 2004, 15(2), 198-208.

33. Guha, M.; Ali, S.Z.; Bhattacharya, S. Twin-screw extrusion of rice flour without a die: effect of barrel temperature and screw speed on extrusion and extrudate characteristics. J. Food Eng. 1997, 32(3), 251-267; DOI: 10.1016/S03+0-8774(97)00028-9.

34. Zavareze, E.D.R.; Dias, A.R.G. Impact of heat-moisture treatment and annealing in starches: A review. Carbohyd Polym. 2011, 83(2), 317-328; DOI: 10.1016/j.carbpol.2010.08.064.

35. Jo, H.R.; Surh, J.H. Effects of cooking methods with different heat intensities on antioxidant activity and physicochemical properties of garlic. J Korean Soc Food Sci Nutr 2016, 45(12), 1784-1791; DOI: 10.3746/jkfn.2016.45.12.1784.

36. Kim, J.S.; Kang, O.J.; Gweon, O.C. Comparison of phenolic acids and flavonoids in black garlic at different thermal processing steps. J. Funct. Foods 2013, 5(1), 80-86; DOI: 10.1016/j.jff.2012.08.006.

37. Yoon, S.R.; Lee, M.H.; Park, J.H.; Lee, I.S.; Kwon, J.H.; Lee, G.D. Changes in physicochemical compounds with heating treatment of ginseng. J Korean Soc Food Sci Nutr 2005, 34(10), 1572-1578; DOI: 10.3746/jkfn.2005.34.10.1572.

38. Kim, M.Y.; Lee, S.H.; Jang, G.Y.; Park, H.J.; Meishan, L.; Kim, S.J.; Lee, Y.R.; Lee, J.S.; Jeong, H.S. Effects of high pressure treatment on antioxidant compounds and activity of germinated rough rice (Oryza sativa L.). J Korean Soc Food Sci Nutr 2013, 42(11), 1783-1791; DOI: 10.3746/jkfn.2013.42.11.1783.

39. Choi, Y.; Lee, S.M.; Chun, J.; Lee, H.B.; Lee, J. Influence of heat treatment on the antioxidant activities and polyphenolic compounds of Shiitake (Lentinus edodes) mushroom. Food Chem. 2006, 99(2), 381-387; DOI: 10.1016/j.foodchem.2005.08.004.

40. Choi, C.S.; Kim, K.I.; Hong, H.D.; Choi, S.Y.; Lee, Y.C.; Kim, K.T.; Rho, J.H.; Kim, S.S.; Kim, Y.C. Phenolic acid composition and antioxidative activity of white ginseng (Panax ginseng, C. A. Meyer). J. Ginseng Res. 2006, 30(1), 22-30; DOI: 10.5142/JGR.2006.30.1.022.

41. Luo, D.; Fang, B. Structural identification of ginseng polysaccharides and testing of their antioxidant activities. Carbohydr Polym 2008, 72(3), 376-381; DOI: 10.1016/j.carbpol.2007.09.006.

42. Wang, J.; Li, S.; Fan, Y.; Chen, Y.; Liu, D.; Cheng, H.; Gao, X; Zhou, Y. Anti-fatigue activity of the watersoluble polysaccharides isolated from Panax ginseng C. A. Meyer. J Ethnopharmacol 2010, 130(2), 421-423; DOI: $10.1016 /$ j.jep.2010.05.027.

43. Kang, S.W.; Min, H.Y. Ginseng, the 'Immunity Boost': the effects of Panax ginseng on immune system. J. Ginseng Res. 2012, 36(4), 354-368; DOI: 10.5142/jgr.2012.36.4.354.

44. Cho, K.L.; Woo, H.J.; Lee, I.S.; Lee, J.W.; Cho, Y.C.; Lee, I.N.; Chae, H.J. Optimization of Enzymatic Pretreatment for the Production of Fermented Ginseng using Leaves, Stems and Roots of Ginseng. J. Ginseng Res. 2010, 34(1), 68-75; DOI: 10.5142/JGR.2010.34.1.068.

45. Han, Y.N.; Kim, S.Y.; Lee, H.J.; Hwang, W.I.; Han, B.H. Analysis of Panax ginseng Polysaccharide by alcian blue dye. J. Ginseng Res. 1992, 16(2), 105-110.

46. Lee, J.W.; Do, J.H. Extraction condition of acidic polysaccharide from Korean red ginseng marc. J. Ginseng Res. 2002, 26(4), 202-205. DOI: 10.5142/JGR.2002.26.4.202.

47. Zhang, X.; Yu, L.; Bi, H.; Li, X.; Ni, W.; Han, H.; Li, N.; Wang, B.; Zhou, Y.; Tai, G. Total fractionation and characterization of the water soluble polysaccharides isolated from panax ginseng C.A. Meyer. Carbohydr. Polym. 2009, 77(3). 544-552; DOI: 10.1016/j.carbpol.2009.01.034.

48. Yang, S.J., Woo, K.S.; Yoo, J.S.; Kang, T.S.; Noh, Y.H.; Lee, J.S.; Jeons, H.S. Change of Korean ginseng components with high temperature and pressure treatment. Korean J. Food Sci. Technol. 2006, 38(4), 521-525.

49. Lee, J.H.; Kwon, K.R.; Cha, B.C. Component analysis of cultivated ginseng, red ginseng, cultivated wild ginseng and red wild ginseng using HPLC method. J Pharmacopuncture 2008, 11(2), 87-95; DOI: 10.3831/KPI.2008.11.2.087. 
50. Yang, D.C.; Yang, K.J.; Choi, Y.E. Production of red ginseng specific ginsenosides (Rg2, Rg3, Rh1 and Rh2) from Agrobacterium - transformed hairy roots of panax ginseng by heat Treatment. J. Photosci. 2001, 8(1), 1922. 